\title{
Quantum interference and imaging using intense laser fields
}

\author{
Kasra Amini ${ }^{1,2}$, Alexis Chacón ${ }^{3,4}$, Sebastian Eckart ${ }^{5}$, Benjamin Fetić ${ }^{6}$, and Matthias Kübel ${ }^{7, a}$ (D) \\ ${ }^{1}$ ICFO - Institut de Ciencies Fotoniques, The Barcelona Institute of Science and Technology, 08860 Castelldefels, Barcelona, \\ Spain \\ 2 Max Born Institute, 12489 Berlin, Germany \\ 3 Department of Physics and Center for Attosecond Science and Technology, POSTECH, 7 Pohang, \\ Pohang 37673, South Korea \\ 4 Max Planck POSTECH/KOREA Research Initiative, Pohang 37673, South Korea \\ ${ }^{5}$ Institut für Kernphysik, Goethe-Universität, Max-von-Laue-Str. 1, 60438 Frankfurt am Main, Germany \\ ${ }_{7}^{6}$ Faculty of Science, University of Sarajevo, Zmaja od Bosne 35, 71000 Sarajevo, Bosnia and Herzegovina \\ 7 Institute of Optics and Quantum Electronics, Max-Wien-Platz 1, 07743 Jena, Germany
}

Received 12 March 2021 / Accepted 7 September 2021 / Published online 20 October 2021 (C) The Author(s) 2021

\begin{abstract}
The interference of matter waves is one of the intriguing features of quantum mechanics that has impressed researchers and laymen since it was first suggested almost a century ago. Nowadays, attosecond science tools allow us to utilize it in order to extract valuable information from electron wave packets. Intense laser fields are routinely employed to create electron wave packets and control their motion with attosecond and ångström precision. In this perspective article, which is based on our debate at the Quantum Battles in Attoscience virtual workshop 2020, we discuss some of the peculiarities of intense light-matter interaction. We review some of the most important techniques used in attosecond imaging, namely photoelectron holography and laser-induced electron diffraction. We attempt to ask and answer a few questions that do not get asked very often. For example, if we are interested in position space information, why are measurements carried out in momentum space? How to accurately retrieve photoelectron spectra from the numerical solution of the time-dependent Schrödinger equation? And, what causes the different coherence properties of high-harmonic generation and above-threshold ionization?
\end{abstract}

\section{Introduction}

Scientific progress has been fueled by the dream to visualize objects or phenomena that are either too small or too fast to be directly perceived by our senses. For example, femtosecond laser pulses offer the opportunity to freeze femtosecond dynamics. This capability has provided unique insights into ultrafast processes such as chemical reactions [1]. Moreover, attosecond electron dynamics have been resolved with the help of short-wavelength attosecond light sources [2-4]. Alternatively, the time resolution can be pushed beyond the femtosecond duration of a laser pulse by using interferometric techniques, e.g., [5-7].

This article is a follow-up to Quantum Battles in Attoscience 2020 virtual workshop and focuses on Quantum Interference and Imaging of molecular structure by means of photoelectrons. The pivotal idea is to image atomic-scale structures by utilizing electrons to overcome the $\sim 1 \mu \mathrm{m}$ diffraction limit of infrared laser light. This is achieved by exploiting the high intensity of the laser pulses to create coherent electron wave pack-

\footnotetext{
${ }^{\mathrm{a}}$ e-mail: matthias.kuebel@uni-jena.de (corresponding author)
}

ets (EWPs) that are driven by the laser field. With their short de Broglie wavelength, electrons allow one to push the spatial resolution to ångström scales, while maintaining the femtosecond (or even attosecond) time resolution dictated by the highly nonlinear light-matter interaction. This concept is shown in Fig. 1. Here, the laser-created electron wave packet diffracts upon recollision [8] with the parent ion, encoding information about the scattering potential.

The unique combination of ultrahigh spatial and temporal resolution makes intense laser pulses extremely attractive for time-resolved imaging. In the present article, we shall focus on techniques that rely on the direct detection of the photoelectrons. The capabilities of this approach can be extended by exploiting highharmonic generation (HHG), which allows one to transfer the favorable properties of the laser-driven electron wave packet into a beam of high-energy photons. The high-harmonic beam can be directly analyzed or utilized in secondary experiments; both approaches have been extremely fruitful but are beyond the scope of the present paper.

The underlying process for the creation of electron wave packets using infrared light is strong-field ioniza- 


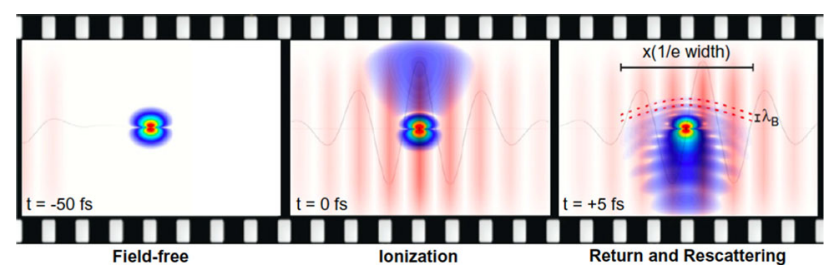

Fig. 1 Quantum mechanical illustration of the creation and rescattering of a photoelectron wave packet in an intense infrared laser field. Calculated using QProp [10]. Figure and caption reproduced from Ref. [11]

tion. Its hallmark feature is the appearance of a series of so-called above-threshold ionization (ATI) peaks in the photoelectron energy spectrum, spaced by the photon energy [9]. An intuitive explanation for these peaks is given in the photon picture: in order to overcome the ionization potential $I_{P}$, an atom may need to absorb $n$ photons. Because of energy conservation, this leads to a discrete photoelectron energy $\hbar \omega, E_{0}=n \hbar \omega-I_{P}$. However, if the field contains sufficiently many photons, the atom may also absorb $m(m=0,1,2,3,4, \ldots)$ excess photons, leading to a series of discrete energies, $E_{m}=(n+m) \hbar \omega-I_{P}$. The companion process of HHG can be interpreted in an analogous manner. Here, selection rules require the number of absorbed photons to be odd, such that a comb of only odd photon energies at $E_{m}=m \hbar \omega(m=1,3,5, \ldots)$ is observed.

The ATI peaks can also be understood in the wave picture as a result of quantum interference: at each field maximum an EWP is created and driven by the laser field. All wave packets will eventually interfere on the photoelectron detector. Because of the field periodicity $T=\frac{2 \pi}{\omega}$, and because time and energy are conjugate quantities, this interference can be observed in the photoelectron energy spectrum as a modulation with periodicity $\hbar \omega$. Incidentally, the HHG peaks can be interpreted to be a result of quantum interference, as well. In this case, the EWP is driven back to parent ion, and recombination leads to the emission of a photon burst [8]. Since equivalent recollisions occur twice per laser cycle, interference of the photon bursts spaced in time by $T / 2$ leads to a modulation of $2 \hbar \omega$ in the high harmonic energy spectrum. Close inspection of the electron dynamics in an intense laser field shows that there exist, in fact, two instances in each laser cycle which yield the same electron drift momentum. This leads to another interference feature, so-called intra-cycle interference whose periodicity varies throughout the photoelectron spectrum $[12,13]$. Importantly, the periodicity of the intra-cycle interference fringes is always larger than $\hbar \omega$, because the difference of the responsible ionization times is always smaller than $T$. Hence, the intracycle interferences create a superstructure on the ATI comb in the energy domain. This raises the questions: if the ATI comb corresponds to photons, what is the corresponding quantity of intra-cycle interferences? Do photons exist on sub-cycle time-scales?
In the past two decades, ATI experiments have progressed from 1D energy-domain to 3D momentumspace measurements. This has been a fruitful path since ATI is rich of interference features in the spatial domain, as well. Analogously to the arguments above, these spatial interference features will manifest in the Fourier domain, i.e., in momentum space. Before we examine such features in detail, we shall discuss in Sect. 2 why measurements are, in fact, conducted in momentum space rather than position space; and, equally importantly, review some techniques used to carry out momentum space measurements, and results obtained therewith.

While measurements are performed in momentum space, our position-space minds desire position space results. In order to retrieve a position space image from a momentum space measurement via Fourier transform, one requires the phase of the momentum wave function, which cannot be directly measured. In Sect. 3, we address the question of how phases can be measured in the laboratory through quantum interference, and discuss an example for reconstructing bound wave functions by holographic interference. By interfering an unknown signal wave with a (known) reference wave, a hologram is created. The concept of holography has been applied to strong-field photoelectron spectroscopy: electron trajectories that scatter from the nucleus (signal) may interfere with trajectories that do not scatter (reference). The resulting hologram, i.e., the interference pattern in the photoelectron momentum distribution may encode information on the scattering potential at the time of rescattering [14-20].

The process of rescattering itself alters quantum interference and encodes structural information of the target. In Sect. 4, we discuss laser-induced electron diffraction (LIED), where an electron wave packet scatters from a molecule to create a diffraction pattern from it. The resulting diffraction pattern can be described as a superposition of the signal resulting from several point-scatterers at the internuclear distance $R$. If the electron wavelength is sufficiently short, the internuclear distance may be retrieved from the diffraction pattern [21]. Moreover, exploiting the intrinsic delay between ionization and rescattering, LIED can be seen as a pump-probe experiment, which has been used to probe nuclear motion not only in diatomic [22] but also polyatomic molecules $[23,24]$. Finally, electron diffraction without rescattering can probe electronic structure [21] and dynamics [25].

For the meaningful interpretation of experiments, it is often essential that experiment and theory go hand in hand. The gold standard in the field of quantum dynamics is the time-dependent Schrödinger equation (TDSE), ideally in all three dimensions [26]. Various implementations of the TDSE have been realized, specifically for the problem of intense light-matter interactions, see, e.g., Ref. [27]. However, a time propagation $\left|\psi\left(t_{f}\right)\right\rangle=U\left(t_{i}, t_{f}\right)\left|\psi\left(t_{i}\right)\right\rangle$ of the multi-dimensional wave function is only half the battle. The other half is retrieving the physical observable of interest from the final wave function. Typically, that is the unbound part 
of the modulus-squared of the momentum-space wave function, i.e., $\left|\phi_{\text {free }}\left(t_{f}\right)\right|^{2}$, representing the photoelectron momentum spectrum. In Sect. 5, we illuminate this particular problem that is imperative for the comparison of experimental and numerical results.

In Sect. 6, we shall discuss the fundamental limitation of all ultrafast imaging methods, namely decoherence. This occurs, for example, in complex physical system where coupling to the environment can lead to the loss of coherence. It is the unspoken necessity of any attempt to resolve quantum dynamics that the dynamics are coherent with the exciting laser field. It is insightful to examine the coherence properties of laser-driven processes. For example, in ATI, the electron wave packets emitted by different atoms do not interfere with each other, i.e., interference takes place on the single-atom level. In HHG, on the other hand, all atoms in the focal volume radiate coherently. What is the underlying reason for this fundamental difference of these closely related effects?

The final chapter is dedicated to recent efforts to expand strong-field physics and related imaging techniques to the condensed phase, particularly quantum materials. These systems can exhibit pronounced coherence effects, and decoherence plays an important role. One key feature of solids, as compared to gases, is the periodicity of the binding potential. This has far-reaching consequences, leading to new quantum mechanical effects to be investigated with the ultrafast imaging toolbox.

Atomic units (a.u.; $\hbar=1,4 \pi \varepsilon_{0}=1, e=1$, and $m_{e}=1$ ) are used throughout the paper, unless otherwise stated.

\section{Position and momentum space}

\subsection{Why measure momentum and not position?}

The position of a bound electron in an atom (e.g., atomic hydrogen) is known to be in the vicinity of its ionic core. Thus, the average momentum of the electron relative to the ionic core must be zero because otherwise the electron would move away from the ionic core and the electron could not be bound. Therefore, the expectation value of the momentum is zero, $\langle\mathbf{p}\rangle=0$. However, the electron possesses non-vanishing kinetic energy, i.e., $\left\langle\mathbf{p}^{2}\right\rangle>0$.

Upon ionization (e.g., because the atom is irradiated with an intense laser pulse), the electron is ejected from the atom and the liberated electron's position coordinate relative to its parent ion changes as a function of time. The liberated electron can be modeled by a wave packet that evolves with time. Since the electron wave packet carries valuable information of the physical system and its dynamics, its characterization is at the very heart of many approaches to study light-matter interaction. This gives rise to an important question: How to characterize the wave function of a freely propagating electron?
Before we answer this question, it is important to be aware that position and momentum are conjugate variables and that the complex-valued wave function's in position space and momentum space are linked by Fourier transformation. This implies that a given electronic state can be fully expressed by using only position space or by using only momentum space coordinates. Despite this equivalence of position and momentum space, there is a fundamental difference comparing momentum and position space when it comes to a freely propagating electron: the momentum of a freely propagating electron is conserved but the position of this electron changes as a function of time. Although this appears to be trivial from a theoretical perspective, it has far reaching consequences regarding the measurement of freely propagating electrons in real experiments.

Let $\Phi(\mathbf{p}, t)$ be the complex-valued electron wave function in momentum space that depends on the time $t$ and the three-dimensional momentum p. In full analogy, $\Psi(\mathbf{x}, t)$ is the complex-valued electron wave function in position space. We exemplify the relationship of position and momentum space wavefunctions for a free electron in Fig. 2. $|\Psi(\mathbf{x}, t)|^{2}$ is time dependent and evolves on ultrafast time scales, whereas $|\Phi(\mathbf{p}, t)|^{2}$ is time independent. This directly shows that the position space distribution has to be characterized as a function of time. In contrast, $|\Phi(\mathbf{p}, t)|^{2}$ is constant. Thus, for a freely propagating wave packet, the expression $|\Phi(\mathbf{p})|^{2}$ is useful without specifying at which time it has been measured.

\subsection{Example: measuring 3D momentum distributions}

Despite the theoretical considerations, how to measure the absolute square of the wave function in momentum space $|\Phi(\mathbf{p})|^{2}$ in real experiments? The state-of-the art method is to use a COLd Target Recoil Ion Momentum Spectroscopy (COLTRIMS) reaction microscope $[28,29]$ which makes use of the dispersion of the wave packet in position space that is shown in Fig. 2. Allowing the wave packets to evolve with time (typically for several nanoseconds instead of several attoseconds as in Fig. 2) in the presence of static external electric and magnetic fields results in a macroscopic distribution of the electron wave packet that has a size of several millimeters when the wave packet hits a time- and position-sensitive detector. (See Fig. 3 for an illustration of a COLTRIMS reaction microscope.) The position and the time are typically measured with a precision of several tens of micrometers and several 100 picoseconds. The additional knowledge about the initial time (typically with a precision on the order of 100 picoseconds) and position (typically with micrometer precision) of the electron in the spectrometer allows for the reconstruction of the three-dimensional momentum distribution $|\Phi(\mathbf{p})|^{2}$ with a resolution of typically $1 / 100$ atomic units. 

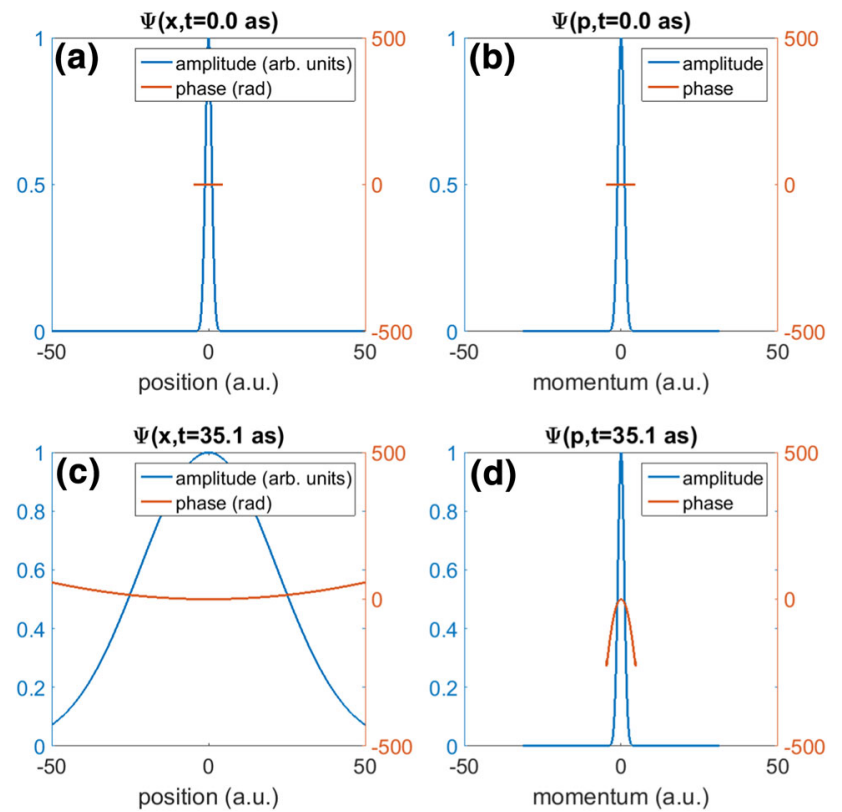

Fig. 2 a shows the wave function $\Psi(\mathbf{x}, t)$ of a Fourierlimited electron wave packet in position space for a given time $t=0$ as. $\mathbf{b}$ shows the same as $\mathbf{a}$ in momentum space. Letting the wave packet evolve for about 35 attoseconds and without applying any forces to the wave packet leads to $\mathbf{c}$ and $\mathbf{d}$, which show the wave function in analogy to a and b. In positions space, dispersion leads to a broadening of the distribution of the amplitudes, whereas the amplitudes in momentum space do not change. Idea taken from a manuscript by H. Schmidt-Böcking, H. J. Lüdde, G. Gruber, S. Eckart and T. Jahnke

This mapping of macroscopic position and time information (nanoseconds and millimeters) to momenta on the atomic scale is shown in Fig. 4. The same conceptual idea underlies the widely used technique of velocity map imaging (VMI) [30]. However, while VMI is similar to COLTRIMS, it (usually) does not resolve the time-of-flight of the particles, resulting in 2D projections of 3D momentum space. However, it should be noted that in fact, both techniques measure velocities and not momenta. Moreover, both techniques can be applied to measure not only electrons, but also ions.

In summary, for the momentum spectroscopy of liberated electrons, it is made use of the fact that the amplitudes in momentum space do not change as a function of time because momentum is conserved for a freely propagating particle.

\subsection{Amplitude information in electron momentum space}

What can be learned from the measured electron momentum distribution $|\Phi(\mathbf{p})|^{2}$ ? One famous example is the idea of the attoclock [33-36]: Using an elliptically polarized single-cycle laser pulse, it is assumed that the most probable time at which the electron starts to tunnel [37] is at the maximum of the laser electric field.

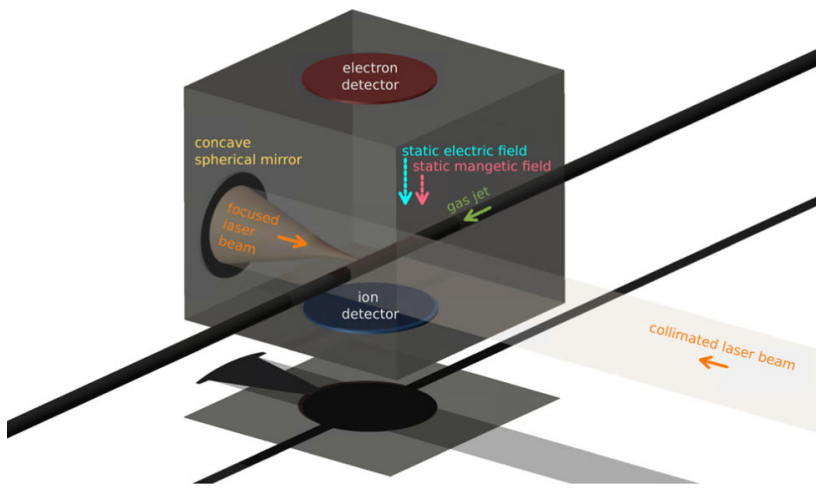

Fig. 3 Basic idea of a COLTRIMS reaction microscope is illustrated. The light-matter interaction occurs in the volume in which the focused laser beam and the gas jet overlap. The homogeneous electric and magnetic fields are used to guide charged particles, which are created in the interaction volume, toward position- and time-sensitive detectors. The gray box highlights the volume in which the charged particles propagate on their way from the interaction region toward the electron or the ion detector. Figure and caption are taken from [31] and have been modified

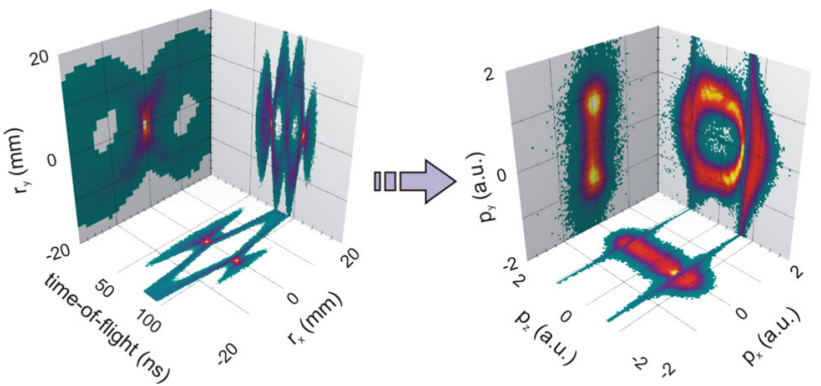

Fig. 4 Three-dimensional detector information (left) can be used to calculate the 3D momentum distribution (right) if the geometry of the COLTRIMS spectrometer and the external electric and magnetic fields are known. The mapping is done by solving the classical equations of motion (making use of the knowledge of the focus position in the chamber, the arrival time of the laser pulse, and the time and position when the detected particle hits the detector). Figure and caption are taken from [32], and the caption has been modified

Further, the final electron momentum is considered to equal the integral of all laser-induced forces acting upon the electron after tunneling. Then, the final electron momentum of the electron can be used to retrieve the time at which the electron appeared at the exit of the tunnel. By evaluating the rotation of the final momentum distribution with respect to the polarization ellipse, the attoclock has been used to investigate the time the electron spends inside the tunnel. This interpretation has led to an ongoing debate [38], also because of conceptional difficulties regarding the bound part of the electron wave function [39], non-adiabaticity [40] and the long-range Coulomb interaction of the electron and its parent ion [41]. Recent experiments have set an upper limit of 1.8 attoseconds to the tunneling 
delay time that is measured by the attoclock upon the strong field ionization of atomic hydrogen [36]. Further examples that study light-matter interaction by interpreting amplitudes in final momentum space are found in Refs. [25, 42-45].

\subsection{Momentum space imaging of electronic orbitals}

Even in the absence of rescattering, the photoelectron momentum distribution (PMD) may encode structural information of its origin. The measured far-field photoelectron momentum distribution can be understood as a diffraction image of the source. Thus, in principle, it should be possible to retrieve structural information by analyzing the diffraction pattern. However, the source is not identical to the atomic or molecular orbital from which the electron is removed but rather the corresponding Dyson orbital. In addition, the details of strong-field ionization have a decisive impact. The momentum distribution along the laser polarization is, of course, determined by the time-dependent laser field, leaving the perpendicular momentum distribution for potential imaging applications. These are, however, hampered by a markedly distorting filtering effect of tunnel ionization, which strongly suppresses large momenta in the direction perpendicular to the laser polarization. Specifically, the perpendicular momentum wave function at the tunnel exit $\left(z=z_{\mathrm{ex}}\right)$ is related to the one at the tunnel entrance, i.e., the Dyson orbital $\left(z=z_{\text {in }}\right)$ by $[46,47]$

$$
\Phi\left(p_{x}, p_{y}, z_{\mathrm{ex}}\right) \propto \Phi\left(p_{x}, p_{y}, z_{\mathrm{in}}\right) \cdot \exp \left[-\frac{\left(p_{x}^{2}+p_{y}^{2}\right) \tau_{T}}{2}\right]
$$

where $\tau_{T}=\frac{\sqrt{2 I_{P}}}{\mathcal{E}}$, and $\mathcal{E}$ is the electric field strength.

The decisive influence of the tunnel filter function (1) makes it necessary to eliminate it in order to retrieve a useful orbital image. This has been achieved in Refs. $[21,48]$ by directly comparing PMDs recorded for parallel and perpendicular alignment, respectively. The difference images reveal clear structures that demonstrate that the PMDs recorded with linear laser polarization contain a filtered projection of the orbitals.

Notably, circular polarization can also be used to map out the orbital shape in combination with molecular orientation [49-51]. This approach, sometimes called "laser STM" (as in scanning tunneling microscope) is similar to the attoclock. Here, however, the unique mapping between the direction of tunneling and drift momentum is exploited to map out the angle dependence of the tunneling probability of aligned or oriented molecules. The partial Fourier transform method $[46,47]$ explains how perpendicular PMD and angledependent tunnel ionization yields are related to the orbital shape. The laser-STM technique has been utilized to resolve angular correlations in sequential double ionization due to a spin-orbit wave packet in Neon cations [52].

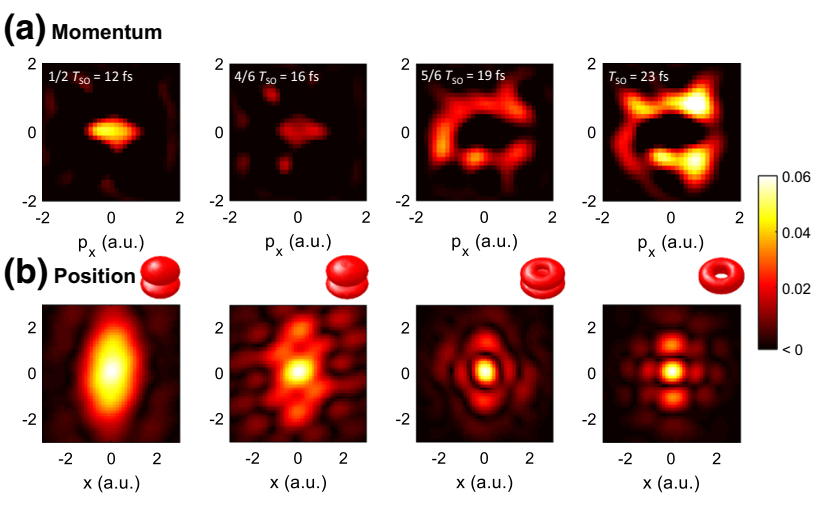

Fig. 5 Snapshots of an electron wave packet in the argon cation obtained by photoelectron orbital imaging. a Momentum-space images recorded at various fractions of the spin-orbit period $T_{\mathrm{SO}}$. The colorbar indicates the visibility of the delay-dependent variations in the recorded PMD. b Position-space images obtained by Fourier transform, assuming a flat phase in momentum space. The expected circular symmetry is broken by a stretch along $p_{x}$, due to an experimental artifact. The figure is adapted from Ref. $[25]$

Direct imaging of a spin-orbit wave packet in $\mathrm{Ar}^{+}$ has been recently achieved by combining tailored laser fields [25]. The delay-dependent PMDs recorded in coincidence with doubly charged ions deliver a movie of the electron motion shown in Fig. 5. In the experiment, a few-cycle pump pulse is used to ionize neutral Ar, producing $\mathrm{Ar}^{+}$in a coherent superposition of two spinorbit states. This causes the vacancy in the $\mathrm{Ar}^{+}$valence shell to oscillate between the $m=0$ and $|m|=1$ states with a period $T_{\mathrm{SO}}=23.3 \mathrm{fs}$, modulating the spatial electron density. The momentum space signatures of these modulations are seen in the experimental snapshots shown in Fig. 5a. After completion of a half-period $1 / 2 T_{\mathrm{SO}}$, the vacancy is in the $|m|=1$ state, and the $m=0$ state, aligned with the laser polarization, is occupied by two electrons. At these delay values, the measured electron density in momentum space exhibits a small spot in the center of the momentum distribution. For alignment of the vacancy in the $|m|=0$ state, at $T_{\mathrm{SO}}$, a ring-shaped electron density is observed. The ring shape can be understood as an image of the donutshaped $|m|=1$ orbital, while the spot in the center relates to the peanut-shaped $m=0$ orbital.

Figure $5 \mathrm{~b}$ shows the spatial images obtained by Fourier transform of the measured momentum space distributions, assuming a flat phase. These spatial distributions do not correspond to the actual spatial orbitals but rather to their autocorrelation signals. The discrepancy with respect to the actual orbitals is most clearly seen for the donut-shaped distribution, which should not be filled in the center. This illustrates how phase information is crucial to reconstruct the spatial orbitals. 


\subsection{Phase information in electron momentum space}

Unfortunately, phases cannot be measured directly, and experimentally only $|\Phi(\mathbf{p})|^{2}$ is accessible (see Sect. 3). The phase of a wave packet in final momentum space is relevant if this wave packet is superimposed with a second wave packet which leads to interference. Here, the absolute phase of the two wave packets does not change the observable quantities, and it is the relative phase of the two wave packets that determines if interference is constructive or destructive. A few examples illustrating the relevance of relative phases in momentum space are briefly described below.

The interference of two electron wave packets that emerge from two different points in position space, e.g., the atoms in a diatomic molecule, can act like a double slit which gives rise to the well-known interference pattern in momentum space for such a two-path interference $[53,54]$. As for a macroscopic double slit also here, the slit geometry defines the observed interference pattern.

ATI for a multi-cycle laser pulse leads to discrete values for the electron energy that can be explained as a consequence of energy conservation or by an inter-cycle interference [13]. The time-dependent light field acts as a grating in the time-domain which is defined by the frequency of the photons. This gives rise to interference in the energy-domain and the spacing of the peaks in the energy spectrum is proportional to the photon energy.

Sub-cycle interference occurs if two wave packets, which overlap in momentum space, are released at times that differ by a timespan that is smaller than the duration of an oscillation of the light field. Conceptionally, sub-cycle interference is very similar to inter-cycle interference $[13,55,56]$. Examples that use sub-cycle interference are two-color attoclock interferometry $[57,58]$ and holographic angular streaking of electrons $[59,60]$. Interestingly, such approaches allow for the access of changes of the Wigner time delay in strong field ionization. The Wigner time delay is the derivative of the phase of the electron wave packet with respect to energy $[61,62]$. However, the modeling of sub-cycle interference by interference of electron wave packets that are born within less than one cycle of the laser field raises the question: Is it possible to model sub-cycle interference (as in Refs. $[13,56,63]$ ) in the energy domain or is the energy picture not suitable to model subcycle processes? Examples toward such a description are found in Refs. [64,65]. However, in order to calculate the coherent sum of all possible pathways in the energy domain, the need for the inclusion of all the corresponding phases and amplitudes of these pathways leads to a very high complexity.

Finally, laser-driven electron recollision leads to various types of interference and diffraction effects, which we discuss in the following Sects. 3 and 4.
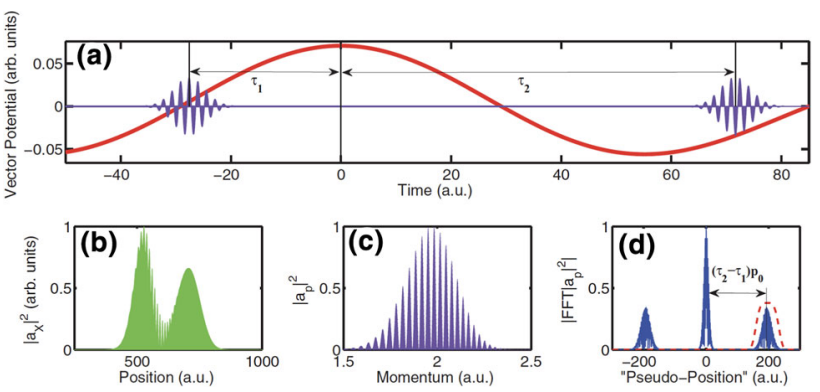

Fig. 6 Schematic representation of QSPIDER. a Depicts: (i) two identical XUV-attosecond pulses (in violet shadow), $\left.\mathbf{E}_{\mathrm{XUV}, 1 / 2}(t)\right)$ with a delay $\tau_{1}$ and $\tau_{2}$ measured with respect to the zero time axis; (ii) In red, the vector potential $\mathbf{A}_{L}(t)$ of the electromagnetic IR laser-field with $800 \mathrm{~nm}$ of wavelength. In b, c, we show the corresponding squared XUVattosecond EWPs for real-space representation (in green) and momentum-space representation in (violet shadows), respectively. The momentum interference in $\mathbf{c}$ is the key in the extraction of the EWP phase difference. $\mathbf{d}$ Fourier decomposition of the momentum EWPs interference $\mathbf{c}$ which clearly has three peaks related to AC components centered at $\tilde{x}_{ \pm}=\left(\tau_{2}-\tau_{1}\right) p_{0}$, obviously $p_{0}$ is the asymptotic EWP central momentum of the $\mathbf{c}$ and an DC component centered at $\tilde{x}=0$. Note, the pseudo-position space is indicated here by $\tilde{x}$. Figure reproduced from Ref. [67]

\section{Quantum interference of electron wave packets}

The interference of two plane waves $\Psi_{1,2}=A_{1,2}$ $\exp \left(i \phi_{1,2}\right)$, where $A$ is the complex amplitude and $\phi$ is the corresponding phase, yields probability density

$$
\left|\Psi_{1}+\Psi_{2}\right|^{2}=\left|A_{1}\right|^{2}+\left|A_{2}\right|^{2}+2 A_{1} A_{2} \cos (\Delta \phi),
$$

which allows access to the relative quantum phase $\Delta \phi=\phi_{1}-\phi_{2}$, and thus to the natural space-time scales (atomic scales, ångström or nanometer and attosecond time) of electron dynamics.

In a next step, we review Quantum Spectral Phase Interferometry for Direct Electron wave-packet Reconstruction (QSPIDER), which is introduced in Refs. $[66,67]$. In the experiment [66], EWPs are created by photoionization of atoms using an attosecond pulse train with a synchronized IR laser field, which induces a momentum shear to the EWP. Due to the periodicity of HHG, the sign of the momentum shear alternates between positive and negative for adjacent pulses. The resulting interference patterns allows for the reconstruction of the EWP's phase, and operates in close analogy to Spectral Phase Interferometry for Direct Electricfield Reconstruction (SPIDER) [5] in optical metrology.

Figure 6 shows the four steps toward the recuperation of a single EWP phase and its amplitude. In a first step, it is assumed that two identical EWPs are produced by replica of same extreme ultraviolet (XUV) attosecond pulse with the simple difference that there exists a time delay between them, as shown in Fig. 6a. The XUV- 
atom interaction at low intensities $\left(10^{10}-10^{12} \mathrm{~W} / \mathrm{cm}^{2}\right)$ and for photon energies $\omega_{\mathrm{X}}>I_{p}$ exceeding the ionization potential, $I_{p}$, is described by perturbation theory. In the second QSPIDER step, a spectral shear is induced in the EWP by means of the weak infrared (IR) laser-field.

In the third step, the final momentum-space distribution is calculated. If the attosecond pulse duration is much shorter than an optical cycle of the IR pulse, $T_{0}$, it can be expressed as the product of an amplitude and, importantly, a phase factor. For an attosecond pulse centered at $\tau_{1}$ with respect to the IR laser, the EWP is described by the following dependencies [67]:

$$
\begin{aligned}
A\left(\mathbf{p}, t_{\mathrm{F}}, \tau_{1}\right) & \propto\left|d\left[\mathbf{p}+\mathbf{A}_{L}\left(\tau_{1}\right)\right]\left(t_{\mathrm{F}}\right)\right| \\
\phi\left(\mathbf{p}, t_{\mathrm{F}}, \tau_{1}\right) & \propto \varphi_{d\left[\mathbf{p}+\mathbf{A}_{L}\left(\tau_{1}\right)\right]}\left(t_{\mathrm{F}}\right)
\end{aligned}
$$

where $d(\mathbf{p})=\mathbf{e}_{x} \cdot \mathbf{d}(\mathbf{p})$ is the $x$-direction component of the dipole transition matrix element $d(\mathbf{p})=$ $\left\langle\mathbf{p}|(-x)| \Psi_{0}\right\rangle$ along the common polarization direction of both the IR laser field and XUV-attosecond pulse, $\left|\Psi_{0}\right\rangle$ is the ground state of the atomic or molecular system, $|\mathbf{p}\rangle$ is the scattering continuum wave, and $-x$ is the dipole moment operator which is proportional to the position operator $x$. Thus, the physical interpretation of $d(\mathbf{p})$ is the complex transition from the ground state $\left|\Psi_{0}\right\rangle$ to the continuum state $|\mathbf{p}\rangle$ mediated by the dipole operator $(-x)$ [68]. In case of a single attosecond EWP, the amplitude in Eq. (3) $A\left(\mathbf{p}, t_{\mathrm{F}}, \tau_{1}\right)$ is proportional to the real amplitude of the dipole transition matrix element $|d(\mathbf{p})|$. In Eq. (4), the dipole phase is $\varphi_{d\left[\mathbf{p}+\mathbf{A}_{L}\left(\tau_{1}\right)\right]}$ which will be extracted as in Ref. [67]. In general, the laser-induced chirp (LIC) generated by the variations of the IR field around the time of ionization needs to be considered. However, in the case of short attosecond pulses ( $<200$ as) and modest intensity $\left(I_{0}<10^{13} \mathrm{~W} / \mathrm{cm}^{2}\right)$, the effects of the LIC phase are negligible. This phase depends on the value of the electric field at ionization time $\tau_{1}$ and is zero if $\mathbf{E}_{L}\left(\tau_{1}\right)=\mathbf{0}$ [67]. It will become relevant for streaking and interferometric measurements as it can become larger than the phase of the dipole.

The most important aspect in this third step is to recover the dipole phase difference $\Delta \varphi_{d\left[\mathbf{p}+\mathbf{A}_{L}\left(\tau_{1}, \tau_{2}\right)\right]}$ which can be approximated as

$$
\Delta \varphi_{d\left[\mathbf{p}+\mathbf{A}_{L}\left(\tau_{1}, \tau_{2}\right)\right]} \approx \frac{\partial \varphi_{d}(p)}{\partial p} \Delta A_{L}
$$

The last step in QSPIDER is to apply the Fourier algorithm to extract the derivative of the dipole phase and integrate it. In the next section, we will follow those steps in $\mathrm{He}^{+}$.

\subsection{Quantum spectral phase interferometry for direct electron wave-packet reconstruction}

The validity of the QSPIDER concept has been verified by a numerical simulation presented in Ref. [67]. To this end, two delayed copies of an EWP with a relative shear between them are used to construct an interferogram, similar to the optical SPIDER technique. This can be realized by focusing an attosecond pulse train (APT) with exactly two pulses centered at $\tau_{1}$ and $\tau_{2}$, onto $\mathrm{He}^{+}$in the presence of a weak IR laser pulse with vector potential $\mathbf{A}_{L}(t)$. One obtains two EWPs which are delayed relative to each other by approximately one optical cycle of the IR laser. The IR laser streaks each of the EWPs resulting in a relative streaking, $\Delta \mathbf{A}_{L}=$ $\mathbf{A}_{L}\left(\tau_{2}\right)-\mathbf{A}_{L}\left(\tau_{1}\right)$, between the two EWPs copies. The streaked and delayed copies produce an interferogram in the final momentum distribution which is conceptually equivalent to the interferogram of the SPIDER technique (see Fig. 6c) [67].

By applying the four steps described in the previous section and the Fourier analysis (see Fig. 6d), we can extract the interesting dipole matrix phase of Eq. (5), which in certain limit is the momentum derivative of the dipole phase. We can also extract the EWP $|A(\mathbf{p})|$ associated with the DC term in the limit $\mathbf{A}_{L}\left(\tau_{1}\right) \approx$ $\mathbf{A}_{L}\left(\tau_{2}\right)$. In the next section, we will apply QSPIDER principles to $\mathrm{He}^{+}$and demonstrate the retrieval of the Dipole matrix elements.

\subsection{Quantum spectral phase interferometry for direct electron wave-packet reconstruction in $2 p$ states}

The two XUV-ATP in the presence of a weak IR laser pulse interacting with $\mathrm{He}^{+}$are shown in Fig. 7. This configuration creates an ideal interferometry scenario to extract the dipole phase derivative and the amplitude.

By applying the Fourier Analysis of SPIDER to the $\mathrm{AC}$ component, the extraction of this dipole phase derivative is shown in Fig. $7 \mathrm{~b}$ and $\mathrm{c}$ for negative and positive p-momentum up to the spectral range which the XUV-pulse allow us. The EWP amplitude has a clear node at $p \sim \pm 1.5$ a.u., as expected for $2 \mathrm{p}$ orbitals. The red and green dots show the QSPIDER reconstruction of the dipole matrix element derivative, which was obtained by dividing the EWP amplitude by the XUVspectral amplitude (see Eq. (3) for the EWP amplitude $\left.A\left(\mathbf{p}, t_{\mathrm{F}}, \tau_{1}\right)\right)$. Concerning the dipole phase reconstruction, we observe clearly a Dirac-like distribution, unique for a system in which the phase has a jump of $\pi$. This jump is shown in Fig. $7 \mathrm{~d}$ and e for positive an negative momenta, and compared to the analytic dipole phase. Good agreement between the reconstruction and the expected dipole phase is found. We also performed TDSE calculation in 1D which are detailed in Ref. [67].

The example of QSPIDER demonstrates how quantum interference of EWPs provides access to phase information of the EWPs, and by extension, of the atomic or molecular system under study. While the first EWP propagates in the continuum, the second one remains bound until the second attosecond pulse arrives, meanwhile probing the system. The information carried by the second EWP is retrieved by considering the first EWP as a known reference wave. This is the concept of holography, and it is applicable to a larger 

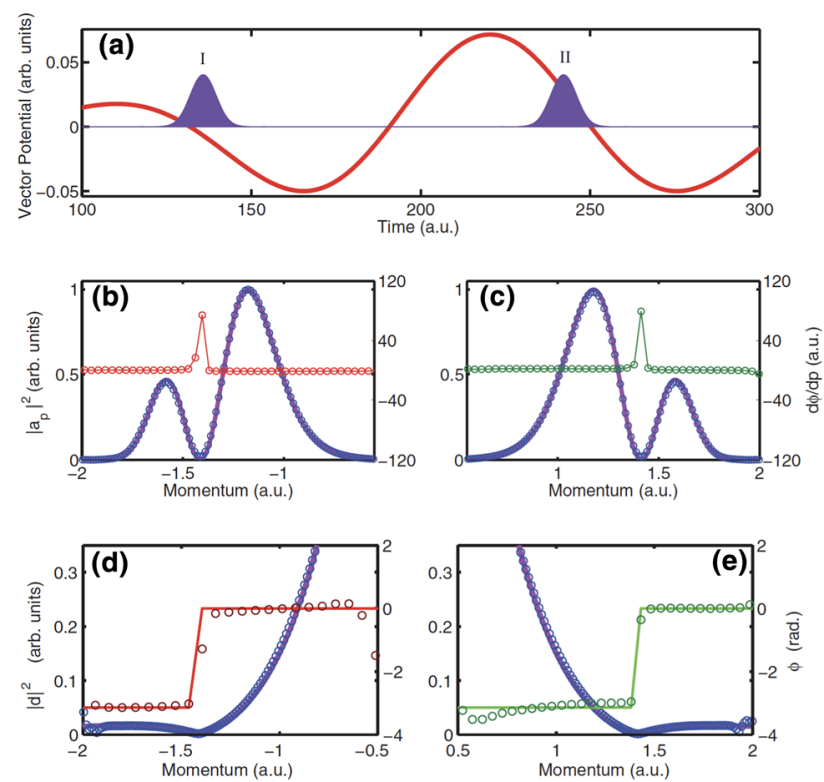

Fig. 7 QSPIDER retrieval for the first excited state of the $\mathrm{He}^{+}$based on strong-field approximation momentum distributions. a The vector potential of the IR laser pulse in red line. The field envelope of the APT in violet areas. The retrieved momentum distribution of the EWP (blue circles) compared to the exact momentum distribution (violet solid line) from the interaction with a single attosecond pulse without IR field is plotted in panels $\mathbf{b}$ and $\mathbf{c}$ for $p<0, \&, p>0$. The retrieved derivative of the dipole phase is shown in red and green circles, respectively, in panels $\mathbf{b}$ and $\mathbf{c}$. The retrieved squared amplitude (blue circles) and phase (red and green circles) of the dipole matrix element is compared to the exact squared amplitude (violet solid line) and phase (red and green solid lines) in panels $\mathbf{d}$ and $\mathbf{e}$

number of experiments in strong field and attosecond physics, in particular to electron rescattering in ATI.

\subsection{Photoelectron holography and its limitations}

When the liberated electron in ATI is driven back to the core, it scatters on the (ionic) potential. In the simplest case of a point-like scatterer, the scattered wave can be approximated as a spherical wave originating at the core. If we consider the unscattered wave as a plane wave and interfere it with the scattered spherical wave, we obtain an interference pattern similar to the one shown in Fig. 8c. It closely resembles the well-known side lobes in the angular distribution of of ATI first reported in Ref. [69], which has been known as holographic interference pattern since the landmark papers from Spanner et al. and Huismans et al. [16,17]. The holographic interpretation of these features allows one to utilize them as a probe of the scattering potential and associated dynamics. Variations in holographic patterns have been used to probe ionization dynamics in two-color experiments [20,55-58,70], molecular dissociation [71], and bound electron and nuclear dynamics [19].

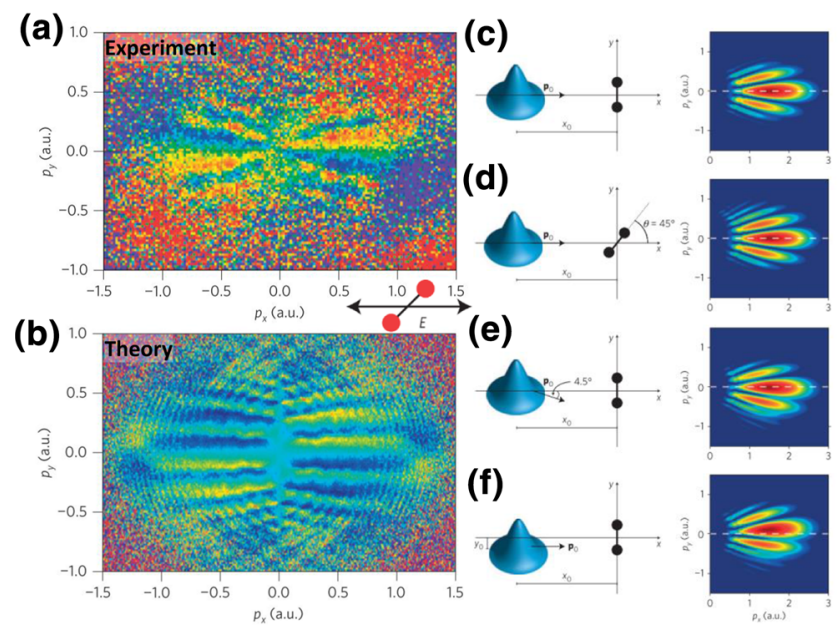

Fig. 8 Photoelectron holography experiment with aligned $\mathrm{N}_{2}$. As indicated by the cartoon in the center of the plot, the data shown in $\mathbf{a}$ is the normalized difference of the PMD recorded for an alignment angle of $45^{\circ}$ and the PMD integrated over all alignment angles. The important feature is the off-center holographic fringe pattern, that is, qualitatively reproduced by the theoretical results shown in $\mathbf{b}$. Panels $\mathbf{c}-\mathbf{f}$ show results of wavepacket scattering simulations for different scenarios: c symmetric reference scenario, d two-center potential set to an angle of $45^{\circ}$, e incident Gaussian wavepacket launched at an angle of $-4.5^{\circ}$, f incident Gaussian wave packet offset in the vertical direction ( $y_{0}=-4$ a.u. $)$. The color scale is logarithmic and covers two orders of magnitude. Figure adapted from Ref. [18] with permission from Nature Publishing Group

However, a word of warning comes from an important paper by Meckel, et al., who studied the effect of molecular alignment on the holographic fringes in PMD [18]. Extending on their pioneering work on LIED [21], the authors carefully varied the angle between the molecular axis and the laser polarization, and found a striking off-center holographic fringe pattern for an angle of $45^{\circ}$ that is reproduced in Fig 8a. This pattern agrees well with results obtained by numerically solving the TDSE, Fig. 8b. For the interpretation of these results, and to understand the meaning of the off-center fringe pattern, simple wave packet scattering simulations are used. These demonstrate that it is not the tilt of the molecular axis that moves the fringes (Fig. 8c, d). It is rather a property of the recolliding wave packet that explains the observations. Specifically, if the wave packet is given a spatial offset relative to the molecular axis, the off-center fringe pattern is obtained (Fig. 8f). This led the authors to conclude that in their "experiment, electron holography provides information about the continuum electron wave packet rather than the scattering object" [18]. This study demonstrates that it is important to know the relevant properties of the recolliding electron wave packet in order to adequately probe molecular structure.

Nevertheless, the example of QSPIDER from Sect. 3.1 shows that recollisions are not a necessary prerequisite for holography. An example that harnesses pho- 
toelectron holography without recollision is holographic angular streaking of electrons (HASE) [59] where a corotating two-color laser field is used to create two electron wave packets that interfere and reveal properties of the phase of the electron wave packet in momentum space. Since for HASE the combined electric field is close to circularly polarized, the continuum wave function, the wave function at the tunnel exit and the bound electron wave function are closely related [60]. This is in contrast to linearly polarized light where recollision and sub-cycle interference lead to a non-trivial relationship of the continuum wave function and the wave function at the tunnel exit $[72,73]$.

\section{Laser-induced electron diffraction}

The process of electron rescattering can lead to interference, even in the absence of an unscattered reference wave. This phenomenon is known as laser-induced electron diffraction (LIED) $[2,11,14,21,22,74-78]$ and is the strong-field variant of ultrafast electron diffraction (UED), whereby a molecule is tunnel ionized to generate an EWP that is used to take a "selfie" of its molecular structure. LIED can retrieve the internuclear distances in a molecule with picometer and attosecond precision.

LIED's extension to the more advantageous midinfrared (MIR, i.e., $\lambda \gtrsim 2 \mu \mathrm{m}$ ) wavelength range has enabled the direct retrieval of many diatomic and more complex molecular structures $[11,23,79-89]$. In fact, not only is the de Broglie wavelength $\lambda_{B}$ of the rescattering electron significantly smaller for a driving laser at $\lambda=$ $3 \mu \mathrm{m}\left(\lambda_{B} \sim 0.75 \AA\right)$ than at $\lambda=0.8 \mu \mathrm{m}\left(\lambda_{B} \sim 2.75 \AA\right)$. The longer driving wavelength also yields a relatively large lateral EWP extent of $\Delta x>150 \AA$ relative to $\Delta x<50 \stackrel{\circ}{A}$ at $\lambda=0.8 \mu \mathrm{m}[11,79,84]$.

Notably, these properties have enabled LIED in the MIR to capture a sub-10-fs snapshot of deprotonation in dissociating $\mathrm{C}_{2} \mathrm{H}_{2}^{2+}$. This was only possible with LIED's sub-optical cycle probe of molecular structure together with its sensitivity to hydrogen scattering. Moreover, ultrafast changes on the rising edge of the LIED pulse have been shown to lead to significant structural deformation in $\mathrm{C}_{60}$ [85], $\mathrm{CS}_{2}$ [82] and OCS [88].

LIED can be well-described using the laser-driven electron-recollision framework [8,90-92] in which the emitted EWP is: (i) accelerated by the oscillating electric field of the intense laser pulse before (ii) returning and (iii) rescattering against the target ion. It is justified to consider only the dominant trajectory that leads to a given drift momentum after rescattering. In the case of LIED, this is the so-called long trajectory, which is produced close to the peak of the electric field. In the quantum mechanical picture of LIED shown in Fig. 1, the emitted EWP is returned and rescattered against two scattering centers (i.e., two atoms in a molecule), leading to interference fringes in the detected electron momentum distribution. These interference fringes are described by the coherent molecular interference term,
$I_{\mathrm{M}}$, which contains structural information and can be in the framework of the independent atom model (IAM) $[75,93,94]$, as given by $[11,95]$

$$
I_{\mathrm{M}}(q) \propto \sum_{i=1}^{N} \sum_{j=1}^{N} f_{\mathrm{i}}(q) f_{\mathrm{j}}^{*}(q) e^{i\left(\mathbf{q} \cdot \mathbf{R}_{\mathrm{ij}}\right)}
$$

Specifically, the phase factor of $I_{\mathrm{M}}$ contains the internuclear distance between two atoms ( $i$ and $j), R_{\mathrm{ij}}$. $I_{\mathrm{M}}$ is a function of the momentum transfer (i.e., $q=$ $\left.2 k_{r} \times \sin \left(\theta_{\mathrm{r}} / 2\right)\right)$ between the incoming EWP and target following scattering, where $f_{\mathrm{i}}$ is the electron scattering amplitude on atom $i$. In fact, $I_{\mathrm{M}}$ is detected as a sinusoidal signal for randomly oriented molecules as given by $[11,95]$

$$
I_{\mathrm{M}}(q) \propto \sum_{i=1}^{N} \sum_{j=1}^{N} f_{\mathrm{i}}(q) f_{\mathrm{j}}^{*}(q)\left(\frac{\sin \left(q R_{\mathrm{ij}}\right)}{q R_{\mathrm{ij}}}\right)
$$

which typically appears as oscillations in the detected momentum distribution of high-energy electrons.

The full three-dimensional momentum distribution of rescattered electrons, as shown in Fig. 9a, can be detected, for example, with a COLTRIMS reaction microscope (ReMi) [28,29,96,97] (also see Sect. 2.2). Importantly, the ReMi can simultaneously detect electrons and ions in kinematic coincidence to select the electron-ion fragmentation channel that is generated during the intense-laser matter interaction in a twostep process. Firstly, the ion of interest (e.g., $\mathrm{H}_{2} \mathrm{O}^{+}$) [86] is identified by selecting its corresponding ion timeof-flight (ToF) range from the ion ToF spectrum, see Fig. 9b. Then, the two-dimensional electron momentum distribution parallel, $p_{\|}$, and perpendicular $p_{\perp}$ of electrons generated with the ion of interest can be generated, see Fig. 9c. Here, the return momentum, $k_{\mathrm{r}}$, at the time of scattering, $t_{\mathrm{r}}$, is obtained by subtracting the vector potential, $A\left(t_{\mathrm{r}}\right)$, of the laser field from the detected rescattered momentum, $k_{\text {resc }}$. The differential cross section (DCS, i.e., number of electrons scattered into a specific solid angle) is extracted by integrating the block arc (yellow) area in Fig. 9c at various different $k_{\mathrm{r}}$.

Figure 9d shows the measured electron yield from ionization of $\mathrm{H}_{2} \mathrm{O}$ [86] for all electrons (blue dashed) and electrons detected in coincidence with $\mathrm{H}_{2} \mathrm{O}^{+}$(black solid) as a function of kinetic energy in units of ponderomotive energy $\left(U_{\mathrm{p}}\right.$, i.e., the cycle-averaged kinetic energy of a free electron oscillating in the electric field of a laser pulse). Figure 9d has two regions clearly distinguishable. Electrons that rescatter (do not rescatter) against the target ion are detected with a typical rescattering kinetic energy of $2-10 U_{\mathrm{p}}\left(0-2 U_{\mathrm{p}}\right)$ and are referred to as "rescattered" ("direct") electrons as indicated by the orange (gray) shaded regions in Fig. 9d. Thus, as a second step, only the $2-10 U_{\mathrm{p}}$ rescattered region is considered for LIED imaging. In this region, one can see that the sinusoidal signal of the $I_{\mathrm{M}}$ is more 


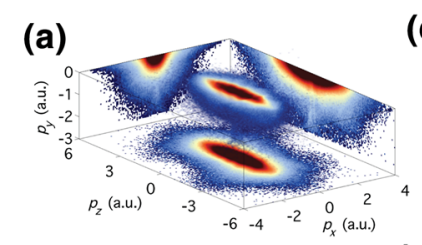

(b)
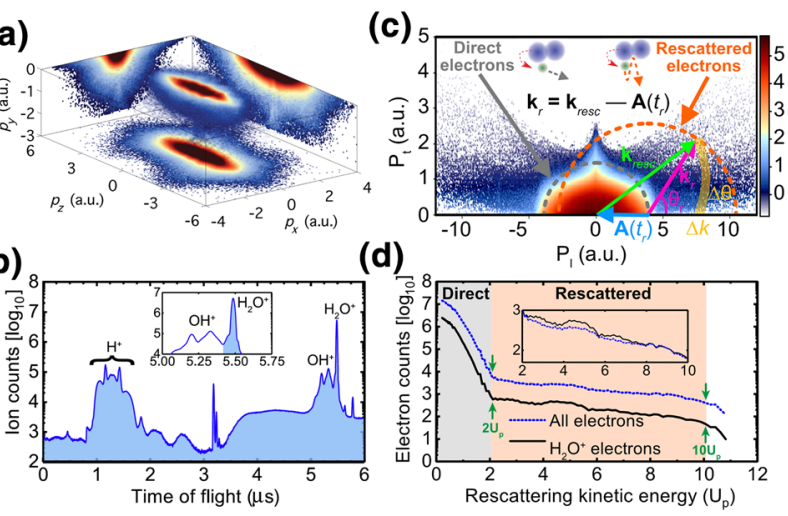

(d)

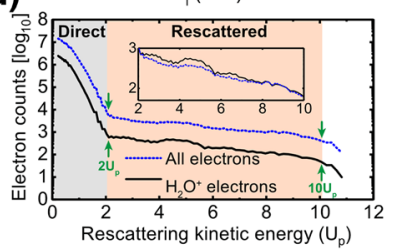

Fig. 9 (LIED rescattered electron data measured with a reaction microscope (ReMi). a Three-dimensional electron data measured with a reaction microscope. [79]. b Typical ion time-of-flight (ToF) spectrum measured from the LIED of $\mathrm{H}_{2} \mathrm{O}$. The inset shows the ToF range corresponding to the most dominant $\mathrm{ToF}$ peak, $\mathrm{H}_{2} \mathrm{O}^{+}$, shaded in blue. c Two-dimensional longitudinal-transverse electron momentum distribution (i.e., $P_{\|}-P_{\perp}$ ). A schematic of typical direct (grey dashed) and rescattered (orange dashed) electron momentum distributions is shown. A diagram defining the relationship between the return momentum, $k_{\mathrm{r}}$, and the vector potential, $A\left(t_{\mathrm{r}}\right)$, at the instance of rescattering, $t_{\mathrm{r}}$, with the detected rescattered momentum, $k_{\text {resc }}$. The DCS is extracted by integrating the electron signal in the area given by the yellow block arc composed of $\Delta \theta$ and $\Delta k$. $\mathbf{d}$ Electron counts as a function of rescattering kinetic energy in units of $U_{\mathrm{p}}$ for all electrons (grey shaded) and $\mathrm{H}_{2} \mathrm{O}^{+}$ electrons (orange shaded). The $2 U_{\mathrm{p}}$ and $10 U_{\mathrm{p}}$ classical cutoffs are indicated by green arrows. The direct and rescattered regions are indicated by gray and orange shaded areas, respectively. Inset shows zoom-in of the rescattered region. Figures adapted from Refs. [79,86]

pronounced in the $\mathrm{H}_{2} \mathrm{O}^{+}$-electron data as compared to the all-electron data. This demonstrates the capability of electron-ion coincidence detection with a ReMi to provide a more sensitive probe of the $I_{\mathrm{M}}$ which would otherwise be washed out by background signal without coincidence selection.

In fact, highly-energetic electrons with detected kinetic energies of hundreds-of-eV range (i.e., $U_{\mathrm{p}} \gg 10 \mathrm{eV}$, see Fig. 10a) are required to achieve an appreciable momentum transfer, $q$, to penetrate beyond the valence electron cloud and scatter against the inner-most core electron shell close to the nuclei. To achieve the high kinetic electron energies, long wavelength driver sources (i.e., $\lambda>2 \mu \mathrm{m}$ in the mid-infrared range) are needed to drive LIED experiments. Extracting the molecular interference signal $I_{\mathrm{M}}$ from the total interference signal $I_{\mathrm{T}}$ requires the subtraction of the background atomic $I_{\mathrm{A}}$ signal. This can be achieved by either calculating the $I_{\mathrm{A}}$ signal using the IAM or by applying a background empirical fit to the detected DCS signal, the latter of which is shown in Fig. 10a. Doing so allows the $I_{\mathrm{M}}$ molecular signal to be contrasted against the $I_{\mathrm{A}}$ atomic signal through the molecular contrast factor,
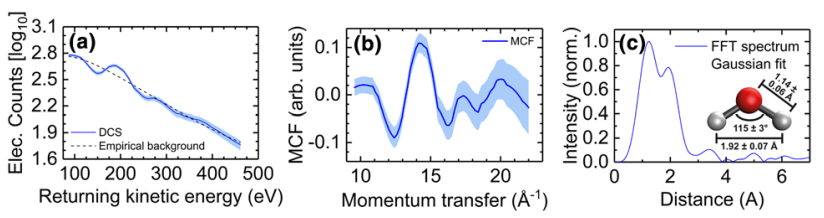

Fig. 10 FT-LIED retrieval of $\mathrm{H}_{2} \mathrm{O}^{+}$molecular structure. a DCS (blue solid) and empirical background (black dashed) as a function of returning kinetic energy. Uncertainty in DCS is shown as blue shaded area. $\mathbf{b}$ MCF as a function of momentum transfer. c Radial distribution following the Fourier transform of panel b. Figure adapted from Ref. [86]

MCF, as given by $[11,95]$

$$
\mathrm{MCF}=\frac{I_{\mathrm{T}}-I_{\mathrm{A}}}{I_{A}}=\frac{I_{\mathrm{M}}}{I_{\mathrm{A}}} .
$$

Figure 10b shows the MCF as a function of momentum transfer, which provides a unique fingerprint of the molecular structure through the sinusoidal signal that is related to the $I_{\mathrm{M}}$. Fourier-transforming (FT) the MCF signal provides the one-dimensional radial distribution of internuclear distances that are present in the molecule. In this case for the LIED imaging of $\mathrm{H}_{2} \mathrm{O}$, two FT peaks are clearly present that correspond to the $\mathrm{O}-$ $\mathrm{H}$ and $\mathrm{H}-\mathrm{H}$ internuclear distances at 1.14 and $1.92 \AA$ when comparing to the literature values. [86]

There in fact exist two variants of LIED, as shown in Fig. 11a: (i) FT-LIED [11,80] or also called fixed-angle broadband laser-driven electron scattering (FABLES) [78], and (ii) LIED based on the quantitative rescattering (QRS) model [75,98], referred to as QRS-LIED. In FT-LIED, the energy dependence of rescattering in back-rescattered electrons are only considered (i.e., varied $k_{r}$ at fixed $\theta_{r} \approx 180^{\circ}$ ). Here, the far-field detected electron momentum distribution can be related to the near-field image of the molecular structure through a FT relation. In QRS-LIED, only the angular dependence of rescattering (i.e., varied $\theta_{r}$ at fixed $k_{r}$ ) is considered at various fixed $k_{r}$ enabling the measurement of the doubly differential cross section (DDCS) of elastic scattering. Figure $11 \mathrm{~b}$ shows the angular dependence of scattering in $\mathrm{N}_{2}$ measured with LIED (blue squares) and with field-free conventional electron diffraction (CED; red line). [22] The very good agreement between LIED and CED demonstrates LIED's ability to extract field-free DCS from field-dressed measurements which are comparable to those measured with CED. Moreover, LIED's sensitivity to hydrogen scattering is demonstrated by its structural retrieval of many hydrogen-containing molecules such as in the deprotonation of $\mathrm{C}_{2} \mathrm{H}_{2}^{2+}$ (see Fig. 11c), as well as in $\mathrm{C}_{2} \mathrm{H}_{2}, \mathrm{H}_{2} \mathrm{O}, \mathrm{NH}_{3}, \mathrm{C}_{6} \mathrm{H}_{6}$ and more [11]. This is particularly pronounced at scattering angles other than forward scattering (i.e., $\theta_{r}>10^{\circ}$ ) where the scattering amplitude of hydrogen scattering is within an order of magnitude of carbon scattering in LIED due to the low kinetic energies of the rescattering LIED electron (see 

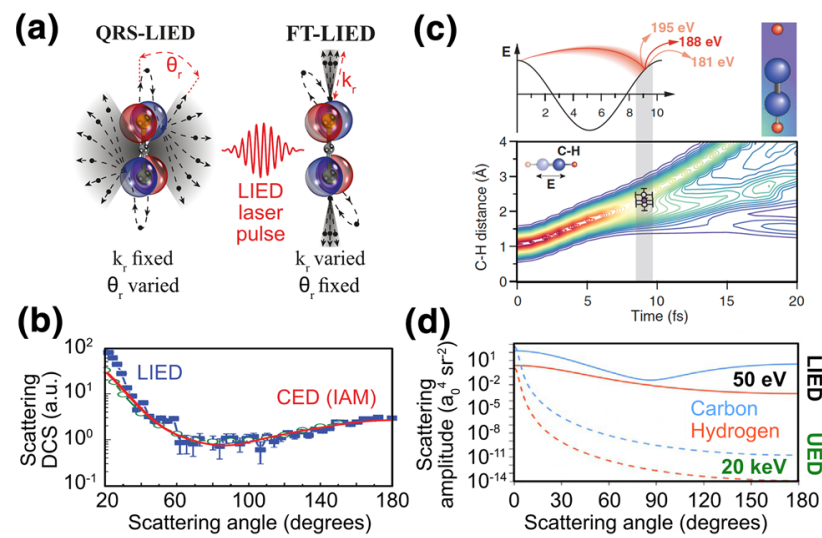

(d)

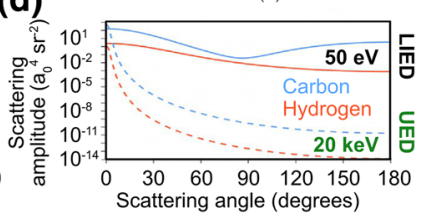

Fig. 11 Variants and characteristics of LIED. a Schematic of QRS-LIED and FT-LIED. b Scattering DCS as a function of scattering angle. LIED (blue squares) and CED data (red line) are shown. c Extracted C-H internuclear distance as a function of time after ionization following the LIED imaging of $\mathrm{C}_{2} \mathrm{H}_{2}^{2+}$. $\mathbf{d}$ Scattering amplitude as a function of scattering angle for carbon (blue) and hydrogen scattering (orange) at typical LIED (solid) and UED (dashed) electron kinetic energies of $50 \mathrm{eV}$ and $20 \mathrm{keV}$, respectively. Figures adapted from Refs. [22, 23, 79, 84]

Fig. 11d). While in UED, the scattering amplitude of hydrogen and carbon scattering at $\theta_{r}>10^{\circ}$ is ordersof-magnitudes lower than in LIED owing to the significantly higher electron kinetic energies used in UED. Although UED is limited to forward-scattering only, time-resolved UED studies have demonstrated to be a very sensitive and powerful probe of molecular structure and photoinduced molecular dynamics using sub-150fs MeV UED electron pulses [11,99-101]. A variety of complementary aspects between field-dressed LIED and field-free UED measurements exist, with many future opportunities to study a variety of gas-phase molecular structures and associated dynamics [11].

\section{How to extract photoelectron momentum distribution from the ab initio calculations?}

Over the last three decades numerical solutions of the time-dependent Schrödinger equation within the singleactive electron (SAE) approximation have emerged as one of the main theoretical tools used to study photoionization and strong-field phenomena. Due to the "black-box" nature of the TDSE, however, the underlying physics are often interpreted using alternative theoretical models [26] and approaches such as the strong-field approximation (SFA, for a review see Refs. $[27,102])$. Other approaches, which are often variants of the SFA, include quantum-orbit theory [103-106], Coulomb-corrected SFA [107,108], Coulomb quantumorbit strong field approximation [109-111], semiclassical two-step model [112-114], classical trajectory based
Monte Carlo method [115-117], quantum trajectorybased Monte Carlo method [118] and many more.

The majority of these methods have one aspect in common, namely they use a trajectory-based picture to describe the field-induced ionization process and the associated electron motion. Importantly, there may exist many different pathways for an electron to reach the detector with the same final linear momentum. Trajectory-based methods allow us to explain features in the photoelectron momentum distribution as quantum interference of different pathways, yielding an intuitive physical interpretation of the photoionization process, which may not be readily available from a TDSE solution. Another advantage of these models is that they are often computationally much simpler than the numerical solution of the TDSE.

The advantage of solving the TDSE, on the other hand, is that it is the most rigorous tool that theorists use to predict and to validate experimental results (see for example Refs. [17,119-123]) and to compare with the predictions of above mentioned methods (see for example Refs. [109-111,124-127]). In this sense, numerical solutions of the TDSE are often used as a benchmark.

In this section, we give a brief introduction to the numerical method for solving the TDSE within SAE approximation and dipole approximation (for more details see Ref. [128]) and give guidelines how to extract PMD from the time-dependent wave packet calculations.

The initial state used as a starting point in the TDSE calculations is obtained by solving the stationary Schrödinger equation for an arbitrary spherically symmetric binding potential $V(\mathbf{r})=V(r)$ in spherical coordinates:

$$
H_{0} \psi(\mathbf{r})=E \psi(\mathbf{r}), \quad H_{0}=-\frac{1}{2} \nabla^{2}+V(r) .
$$

The solution $\psi(\mathbf{r})$ can be written as

$$
\psi_{n \ell m}(\mathbf{r})=\frac{u_{n \ell}(r)}{r} Y_{\ell}^{m}(\Omega), \quad \Omega \equiv(\theta, \varphi)
$$

where the $Y_{\ell}^{m}(\Omega)$ are spherical harmonics. The radial function $u_{n \ell}(r)$ is a solution of the radial Schrödinger equation:

$$
\begin{aligned}
& H_{\ell}(r) u_{n \ell}(r)=E_{n \ell} u_{n \ell}(r), \\
& H_{\ell}(r)=-\frac{1}{2} \frac{d^{2}}{d r^{2}}+V(r)+\frac{\ell(\ell+1)}{2 r^{2}},
\end{aligned}
$$

where $n$ is the principal quantum number and $\ell$ is the orbital quantum number. The initial wave function $\psi_{n \ell m}(\mathbf{r})$ is propagated under the influence of an intense laser field as described by the TDSE:

$$
i \frac{\partial \Psi(\mathbf{r}, t)}{\partial t}=\left[H_{0}+V_{I}(t)\right] \Psi(\mathbf{r}, t)
$$


where $V_{I}(t)=-i A(t) \partial_{z}$ is the interaction operator in the dipole approximation and velocity gauge.

We assume that the laser field is linearly polarized along the $z$ axis, so that the vector potential is given by $A(t)=-\int^{t} E\left(t^{\prime}\right) d t^{\prime}$, where $E(t)$ is the electric field of the laser pulse:

$$
E(t)=E_{0} \sin ^{2}\left(\frac{\omega t}{2 N_{c}}\right) \cos (\omega t), \quad t \in\left[0, T_{p}\right]
$$

Here, $E_{0}$ is the electric field amplitude, $\omega=2 \pi / T$ is the laser-field frequency and $T_{p}=N_{c} T$ is the pulse duration, with $N_{c}$ the number of optical cycles. At the end of the laser-atom interaction $t=T_{p}$, we obtain the time-dependent wave function $\left|\Psi\left(T_{p}\right)\right\rangle$ which contains all relevant information about the simulated process.

The question is how do we extract this information from the final wave function $\left|\Psi\left(T_{p}\right)\right\rangle$ ? The formally exact PMD can be extracted from $\left|\Psi\left(T_{p}\right)\right\rangle$ by projecting it onto the continuum states of the field-free Hamiltonian $H_{0}$ having the linear momentum $\mathbf{k}=\left(k, \Omega_{\mathbf{k}}\right)$, $\Omega_{\mathbf{k}} \equiv\left(\theta_{\mathbf{k}}, \varphi_{\mathbf{k}}\right)$. We call this method the PCS (Projection onto Continuum States) method. In a typical photoionization experiment, a photoelectron ends up in quantum states with a linear momentum $\mathbf{k}$, so that corresponding continuum states must be localized in momentum space. The continuum states that describe such a quantum state obey the so-called incoming boundary condition and can be written as the partialwave expansion $[129,130]$ :

$$
\psi_{\mathbf{k}}^{(-)}(\mathbf{r})=\sqrt{\frac{2}{\pi}} \frac{1}{k} \sum_{\ell, m} i^{\ell} e^{-i \Delta_{\ell}} \frac{u_{\ell}(k, r)}{r} Y_{\ell}^{m}(\Omega) Y_{\ell}^{m *}\left(\Omega_{\mathbf{k}}\right),
$$

where $\Delta_{\ell}$ is the scattering phase shift of the $\ell$ th partial wave. The continuum states (15) merge with the plane wave at the time $t \rightarrow+\infty: \psi_{\mathbf{k}}^{(-)}(\mathbf{r}, t) \rightarrow$ $(2 \pi)^{-3 / 2} e^{i\left(\mathbf{k} \cdot \mathbf{r}-E_{\mathbf{k}} t\right)}$. The probability $P\left(E_{\mathbf{k}}, \theta_{\mathbf{k}}\right)$ of detecting the electron with kinetic energy $E_{\mathbf{k}}=k^{2} / 2$ emitted in the direction $\theta_{\mathbf{k}}$ is given by

$$
P\left(E_{\mathbf{k}}, \theta_{\mathbf{k}}\right)=2 \pi k\left|\left\langle\psi_{\mathbf{k}}^{(-)} \mid \Psi\left(T_{p}\right)\right\rangle\right|^{2},
$$

where $\mathbf{k}=\left(k_{x}, k_{z}\right)=\left(k \sin \theta_{\mathbf{k}}, k \cos \theta_{\mathbf{k}}\right)$.

It is worthy to note that in some cases it can be cumbersome to obtain continuum states since the continuum states are only known in an analytical form for the pure Coulomb potential. If that is the case, an approximate PMD can be obtained by what we call the PPW (projection onto plane waves) method. This approach for obtaining the PMD from the time-dependent wavepacket calculations has been introduced and discussed in detail in Ref. [131].

After the laser field has been turned off, the wave function $\left|\Psi\left(T_{p}\right)\right\rangle$ is propagated for a time $\tau$ under the influence of the field-free Hamiltonian $H_{0}$. The time

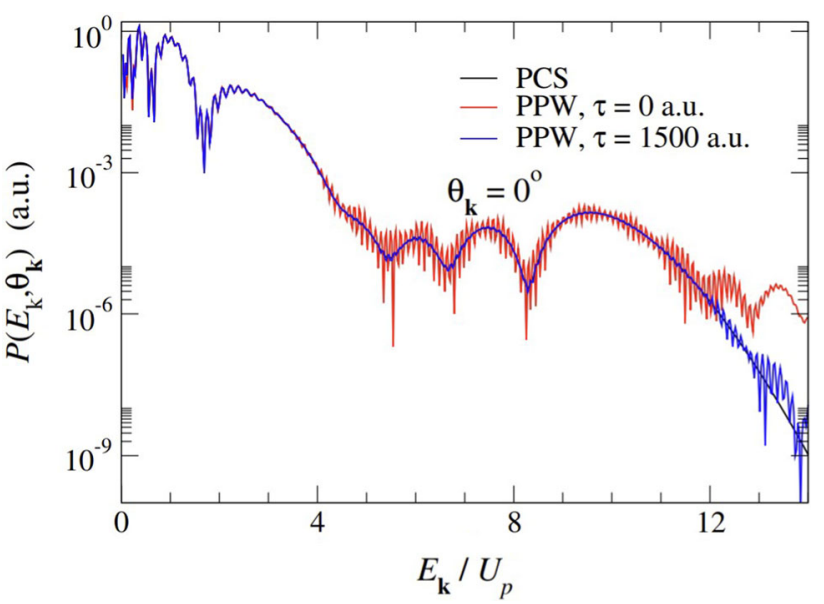

Fig. 12 Photoelectron spectra for fluorine negative ion $\mathrm{F}^{-}$ obtained by the PCS and PPW methods for $\theta_{\mathbf{k}}=0^{\circ}$. The exact PCS spectrum is depicted with the solid black line, while results obtained by the PPW method are depicted by the red and blue solid lines obtained with two different postpulse propagation times as indicated in the legend. The laser field parameters are $I=1.3 \times 10^{13} \mathrm{Wcm}^{-2}, \lambda=1800 \mathrm{~nm}$, and $N_{c}=4$

interval $\tau$ has to be large enough so that even the slowest part of the wave function $\left|\Psi\left(T_{p}+\tau\right)\right\rangle$ has reached the asymptotic region $r>R$ where we can neglect the atomic potential, $V(r) \approx 0$. By excluding the bound part of the wave function $\left|\Psi\left(T_{p}+\tau\right)\right\rangle$ which we assume is spatially localized in region $r<R$, we can obtain PMD by projecting the continuum part of the wave function onto a plane wave:

$P\left(E_{\mathbf{k}}, \theta_{\mathbf{k}}\right) \approx P^{\prime}\left(E_{\mathbf{k}}, \theta_{\mathbf{k}}\right)=2 \pi k\left|\left\langle\Phi_{\mathbf{k}} \mid \Psi^{\prime}\left(T_{p}+\tau\right)\right\rangle\right|^{2}$,

where we use plane wave $\Phi_{\mathbf{k}}(\mathbf{r})$ given as the partialwave expansion:

$$
\Phi_{\mathbf{k}}(\mathbf{r})=\sqrt{\frac{2}{\pi}} \sum_{\ell, m} i^{\ell} j_{\ell}(k r) Y_{\ell}^{m}(\Omega) Y_{\ell}^{m *}\left(\Omega_{\mathbf{k}}\right) .
$$

where $j_{\ell}(k r)$ is the spherical Bessel function of order $\ell$. The prime on the time-dependent wave function in (17) indicates that we take only part of the wave function $\left|\Psi\left(T_{p}+\tau\right)\right\rangle$ that has reached beyond the border of the asymptotic region, $r>R$. In all presented calculations, we have set $R=40$ a.u.

Let us now compare these two approaches for obtaining PMD. As an example, we use the fluorine negative ion $\mathrm{F}^{-}$. This choice is motivated by the fact that after electron emission, there is no long-range (i.e., Coulombic) potential, and hence, the applicability of the PPW method is clear. Within the SAE approximation, we model the corresponding potential by the Green-SellinZachor potential with a polarization correction included [132]: 

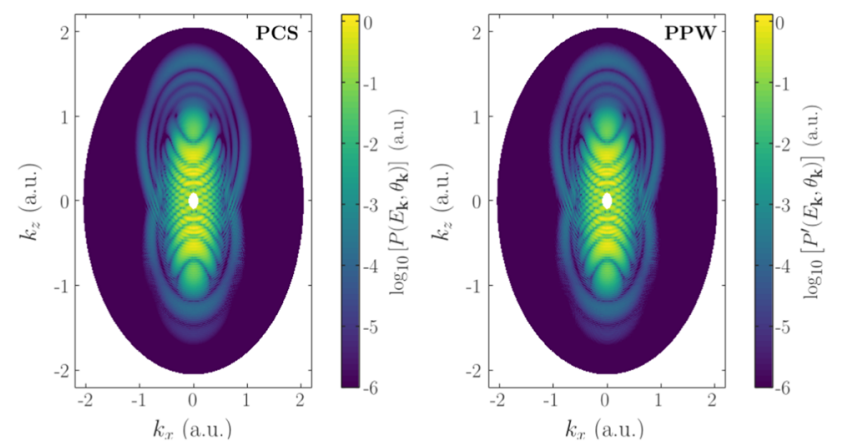

Fig. 13 Full PMD for fluorine negative ion $\mathrm{F}^{-}$obtained by the PCS and PPW methods. The left panel shows PMD obtained by the PCS method and the right panel shows PMD obtained by the PPW with $\tau=1500$ a.u. Laser field parameters are the same as in Fig. 12

$$
V(r)=-\frac{Z}{r\left[1+H\left(e^{r / D}-1\right)\right]}-\frac{\alpha}{2\left(r^{2}+r_{p}^{2}\right)^{3 / 2}},
$$

with $Z=9, D=0.6708, H=1.6011, \alpha=2.002$, and $r_{p}=1.5906$. The $2 p$ ground state of $\mathrm{F}^{-}$has the electron affinity equal to $I_{p}=3.404 \mathrm{eV}$.

In Fig. 12, we show ionization probability in the direction $\theta_{\mathbf{k}}=0^{\circ}$ for the laser field intensity $I=$ $1.3 \times 10^{13} \mathrm{Wcm}^{-2}$, wavelength $\lambda=1800 \mathrm{~nm}$, and four optical cycles laser pulse duration, $N_{c}=4$. The solid black line represent the ionization probability obtained by the PCS method. The red line represent the ionization probability obtained by the PPW method with the post-pulse propagation of the wave function equal to $\tau=0$ a.u. We can see that the low-energy part of the photoelectron spectrum agrees quite well with the exact result. On other hand, the high-energy part of the spectrum exhibits oscillations which are absent in the PCS results. These oscillations can be smoothed by increasing the time of the post-pulse propagation up to $\tau=1500 \mathrm{a} . \mathrm{u}$. The results for $\tau=1500 \mathrm{a} . \mathrm{u}$. are depicted by the blue solid line. Our experience tell us that general rule of thumb is that as we increase the postpulse propagation time, the agreement between these two methods becomes better in the high-energy region, although this implies that we have to use a larger spatial grids on which the TDSE is numerically solved. Therefore, one has to compromise between the consumption of computing resources and obtaining fully converging photoelectron spectrum.

In Fig. 13, we show full PMD obtained by the PCS (left panel) and PPW methods with $\tau=1500$ a.u (right panel). The laser field parameters are the same as in Fig. 12. As we can see, these two methods produce identical PMDs.

In the case of atomic photoionization where the liberated electron moves in the modified Coulomb potential, it would be the natural choice to use the Coulomb waves as a good approximation to the true continuum states, but our calculations show that even in the case of atomic photoionization, the plane waves must be used as the final states of the detected electron since only the plane waves are eigenstates of the momentum operator. Analogous studies for atomic ionization are currently being prepared and will be published elsewhere.

\section{Coherence, decoherence and incoherence}

Coherence is defined as the capability of waves to interfere. The extension to waves of different frequencies is the foundation of mode locking in femtosecond lasers. Interestingly, we encounter coherence in both ATI and HHG, which are characterized by frequency combs of photoelectron or photon energies, respectively. The time-domain flip side of the frequency comb are the "pulse trains" of the continuum EWPs created around the peaks of the laser electric field. The sub-cycle EWPs themselves can be understood as multi-mode interference of coherent electron waves originating from the same laser half-cycle. In the present case of continuum wave packets, the frequency (momentum) spectrum of the wave packets is wide and continuous.

Ultrafast science is particularly interested in tracking the evolution of bound wave packets, as they allow microscopic insights into the dynamics occurring inside atoms, molecules or solids. Typically, bound wave packets have a discrete spectrum, implying a periodicity in time. Strong-field ionization has been shown to allow the preparation of coherences between different electronic states [133]. Well-known examples of bound electron wave packets that have been tracked with ultrahigh time resolution include spin-orbit wave packets in rare-gas ions $[25,52,134]$ and charge migration in polyatomic molecules $[135,136]$. Furthermore, periodic vibrational [137] and rotational wave packets [138-140] in molecules have been tracked.

Contrary to the periodic example considered above, processes such as chemical reactions are typically nonperiodic. In this context, we can consider a process as coherent if its time evolution clearly depends on some initiating event, usually the interaction with a pump laser pulse. This is the prerequisite to study ultrafast dynamics, for example using a pump-probe scheme.

Some processes can lead to the apparent loss of coherence, often referred to as decoherence. This may occur, for example, in the vicinity of conical intersections, where the electron and nuclear degrees of freedom are strongly coupled. Hence, energy stored in electronic degrees of freedom can be transferred to vibrational motion, and the relationship between the pump event and the ensuing dynamics is lost. A particularly interesting example is the charge migration process studied by Callegari, et al. [135]. They observed an oscillating ion yield due to electronic coherence immediately following XUV photoionization of phenylalanine [135]. After a few 10s of femtoseconds, the delay-dependent oscillations disappear and the measured signal became static. This is a clear signature of decoherence. 
Generally, coherence is lost if a coherent system is coupled to a "bath," and it is interesting to scale the size of the bath. For example, consider electron emission from a pair of identical atoms at a fixed internuclear distance $R$. Emission could originate from either one of two atoms, leading to an interference term similar to the one in LIED, $\exp (i k R)$. In this example, the nuclei represent the bath that may be coupled to the electron motion. Kunitski, et al., realized such an experiment using Neon dimers exposed to an intense circularly polarized laser field [54]. They found that sought-after interference can be observed when they keep track of the bath, i.e., measure the nuclei in coincidence with the electrons. Specifically, an interference structure only appears if one selects for the parity of the ionic state on which $\mathrm{Ne}_{2}^{+}$dissociates [54]. Experiments on the dissociative multiphoton ionization of $\mathrm{H}_{2}$ [141] can be interpreted in a similar manner. In the photoelectron spectrum, no clear ATI peaks are seen. However, when considering the energy transferred to the nuclei, the coherent peak structure of ATI is restored. Again, the energy absorbed by the nuclei only seemingly leads to decoherence but coherence is maintained if the measurement includes all relevant observables in the bath.

With increasing complexity, it becomes increasingly difficult to keep full track of the bath. For example, in polyatomic molecules a plethora of nuclear degrees of freedom exists, such that it is extremely challenging to measure all of them once nuclear motion sets in, which happens a few femtoseconds after the pump pulse. This challenge increases even more, if one allows interaction with the environment. An illustrative example is the work of Hackermüller et al., who studied the decoherence of matter waves due to thermal emission of radiation [142]: as the temperature is increased, decoherence becomes stronger due to thermal emission of radiation. Similarly, lasing or superfluorescence are impeded by spontaneous emission or nonradiative transitions [143], while decoherence of molecular rotations occurs through collisions with the environment $[144,145]$. These decoherence phenomena relate to ongoing efforts to test the limits of quantum mechanics by studying interference phenomena in mesoscopic systems [146].

Let us return to the prototypical examples of ATI and HHG of gaseous atoms. Despite the absence of internal degrees of freedom that could lead to decoherence, ATI of different atoms is incoherent, i.e., all interference phenomena in ATI take place on the singleatom level. This is entirely different to the case of HHG where all atoms in the focal volume radiate harmonics coherently. As a consequence, phase matching is an important issue for the case of HHG [147], but irrelevant for ATI. Moreover, this "macroscopic coherence" of the HHG process is the reason for the sharp HHG peaks, while the contrast between ATI peaks is much lower. Given their close relationship, this difference between $\mathrm{HHG}$ and ATI is remarkable. But what is the underlying reason? An important difference between the two processes is the fact that ATI leads to the production of an ion, while the atom has returned to its ground state after HHG. In other words, it is possible to tell which atom has undergone ATI, but not which one has undergone HHG. This information is equivalent to measuring through which slit the particle passes in Young's double slit experiment. This picture agrees well with the Neon dimer experiment discussed above: if we know the ionic state, the interference is restored.

If the created ion destroys the coherence of ATI from multiple sources, this raises the question whether we can make ATI coherent by studying solid-state systems, where no ion is created. Suitable candidates would be extended systems in the condensed phase, perhaps systems of nanostructures [148].

\section{Toward nonlinear ultrafast spectroscopy of quantum materials}

Recently, the HHG in solids has been attracting the attention of condensed matter physics, see [149] for a recent review. The effect can be observed at quite moderate intensities below the ionization threshold. As such it allows probing solid-state sample without inflicting optical damage. HHG may be useful for the study of several transport charge and spin properties, not only in semiconductors, but also in materials which exhibit unique and novel topological effects such as 2D and 3D topological insulators [150-153].

The character of physical laws on the atomic scale (ångström and nanometer) is dramatically different in solids than in gases [153-158]. Specifically, in the SFA, the energy of the ground state is assumed constant with respect to the momentum $\mathbf{p}$, while the energy spectra of the continuum states at large distance from the parent ion can be considered as parabolic in p [154]. In condensed matter terms, the former corresponds to an infinite hole mass. Thus, in gases, the ground state is localized, while the free continuum electron is moving in a trajectory driven by the laser field. Re-combination may take place at the ground state initial position in gases. On the contrary, in solids, the situation is rather different due to periodicity of the static potential. The possibility of electron-electron correlation effects $[159,160]$, electron-phonon effects, spin-orbit couplings [161-163] and spin orders' effects [164] offer a new and extremely attractive research field for ultrafast spectroscopy and laser control. For instance, the hole can be driven by the laser as well, since the energy dispersion of valence states is not zero $[154,155]$. This causes novel ultrafast dynamics, which differ from those in the gas phase, and are present not only in ordinary semiconductors, but also in quantum materials $[157,158,165,166]$.

Specifically, nonlinear spectroscopy has made it possible extracting information of the band structure in semiconductors using a pump-probe scheme and associating the HHG spectra to the inter-band mechanism [153]. Furthermore, ultrafast metrology at THz frequencies has allowed for the observation of electron-hole 


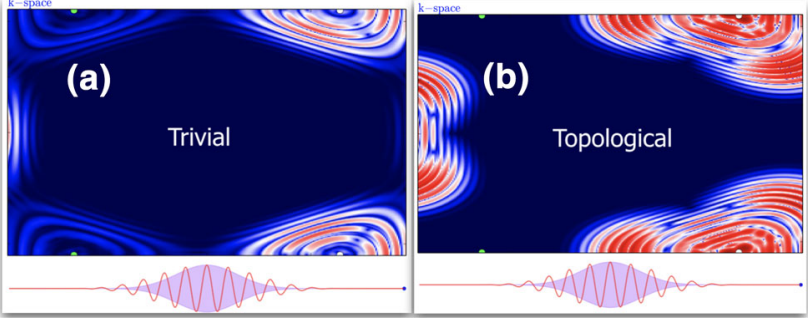

Fig. 14 Quantum interference in trivial and topological materials. Final crystal momentum distribution in Haldane model for the conduction band of the trivial and topological phase, are depicted in $\mathbf{a}$ and $\mathbf{b}$, respectively. Both, trivial and topological materials have the same band gap $\left(\varepsilon_{g} \approx 3.0 \mathrm{eV}\right)$, but exhibit totally different topological invariant or Chern number $\nu=0$, for the trivial phase, $\nu=+1$ for the topological phase. The same circularly polarized driving laser was used for both topological phases

recombination and Bloch oscillation at special valley points in $\mathrm{MoS}_{2}$ or $\mathrm{WS}_{2}[165,167]$. Additionally, attosecond transient absorption experiments for core valence electrons in solids are nowadays in the focus of attention of attosecond condensed matter physics $[167,168]$. The rapidly emerging field of nonlinear spectroscopy in quantum materials [168-170], i.e., topological materials, Weyl semimetals, etc. $[161,166,169,170]$ is attracting the attention of several experimental and theoretical research groups around the world. Those materials are extremely important, since their special features, i.e., topological conducting and isolating bands protected by the fundamental symmetries are robust against energy dissipation and material perturbations $[157,158]$. These unique features promise interesting application of topological insulators (TIs) $[171,172]$ in the optimization of electronic devices, more precisely the transistors and the logical operations defined in the electronic devices $[165,173,174]$.

Attosecond science will expand its frontiers to new challenges, research fields and may open up new options to control transport and optical features in quantum materials [165]. For example, HHG or other nonlinear optical techniques may provide access to the electronic and dynamical properties of quantum materials. The topological invariant defines whether or not a material is topological. It is directly linked to the electron wave function from the crystalline structure, which characterizes the transversal current with respect to a longitudinal applied voltage, exhibiting quantum anomalous Hall effects. The open question which still remain a challenge is how the topological invariants might be associated with the HHG spectrum.

We give here an example from the area of topological ultrafast nonlinear spectroscopy, especially in the interaction of an ultrashort and intense MIR laser wavelength of $3.2 \mu \mathrm{m}$ and intensity of $10^{11} \mathrm{~W} / \mathrm{cm}^{2}$. The Haldane model (HM) is the first which appears to predict quantum Hall effects (QHEs) without Landau levels or more precisely without magnetic fields [175]. This model belongs in the class of the so-called Chern insulator classification for topological materials which simply means the Chern number, $\nu=\frac{1}{2 \pi} \int_{\mathrm{BZ}} d^{2} \mathbf{k} \cdot \boldsymbol{\Omega}(\mathbf{k})$, can be $\nu=-1,0,+1$. HM thus has three topological different phases, i.e., for $\nu= \pm 1$ the system will show QHEs (or transversal quantized conductivity) and, for $\nu=0$ there will not be QHEs. In Ref. [166], a full revision of HM is done in the context of how HHG can encode the topological invariant $\nu$ by means of the circular dichroism, i.e., the produced asymmetry photon emission yield by right- and left-circularly polarized driven lasers, also a parallel work by Silva et al. [169] shows that HHG can be used to track topological phases and transitions in the similar model, but using as an observable, the helicity of the HHG produced by linearly polarized driving lasers.

Recently, Baykusheva et al. has shown numerical results of $\mathrm{HHG}$ from $\mathrm{Bi}_{2} \mathrm{Se}_{3}$, a typical 3D-topological insulator (TI) [163]. In this work, an interesting anomalous enhancement in the nonlinear optical responses of $\mathrm{Bi}_{2} \mathrm{Se}_{3}$ was observed as the driving laser polarization is varied from linear to circular [166]. A theoretical method was developed, which splits the contributions from the topological surface states and bulk surface states, indicating that the responsible mechanism of that enhancement is the spin-orbit couplings of the surfaces states.

In another example in Fig. 14, we present the final crystal momentum in the first Brillouin zone for two different topological phases $\nu=0$ and $\nu=+1$, respectively. The green and white points denote the $\mathrm{K}^{\prime}$ and $\mathrm{K}$ points, respectively. The final interferogram patterns are specially different at K-points for the topological phase with a thinner fringes for the topological phases than the trivial one.

This shows that the interesting topological features can be contained by the final momentum distribution of the conduction bands. Note however, the question of how to measure this distribution inside the material and how to extract the topological invariant $\nu$ are still open for ultrafast sciences and also condensed-matter physics. In particular, and in a broad sense in quantum materials [165] such as Dirac and Weyl semimetals [176] in which two different and opposite Chern numbers lead to the generation of Fermi arcs or pseudomagnetic mono-poles or Weyl-fermions with chirality features [176]. Theoretical and experimental methods, that allow to extract such information based on ultrafast light-matter interaction, will have to be developed in the future.

Acknowledgements The authors thank B. Cooper, A. Maxwell, and C. Faria for organizing Quantum Battles in Attoscience, and M. Vrakking for his valuable suggestions. K.A. acknowledges financial support from the European Research Council (788218) and the Polish National Science Center within the project Symfonia (2016/20/W/ST4/ 00314). A.C. acknowledges Max Planck POSTECH/KOREA Research Initiative Program [Grant No. 2016K1A4A4A01922 028] through the National Research Foundation of Korea (NRF) funded by Ministry of Science, ICT \& Future Planning, partly by Korea Institute for Advancement of Technology (KIAT) grant funded by the Korea Govern- 
ment (MOTIE) (P0008763), The Competency Development Program for Industry Specialist. S.E. and M.K. acknowledge funding by the German Research Foundation (DFG) through priority program SPP 1840 QUTIF. B.F. acknowledges support by the Ministry for Education, Science and Youth, Canton Sarajevo, Bosnia and Herzegovina. M.K. acknowledges funding by the DFG under Project No. 437321733.

\section{Author contributions}

All authors contributed to the preparation of the manuscript. All authors have read and approved the final manuscript.

Funding Open Access funding enabled and organized by Projekt DEAL.

Data Availability Statement This manuscript has no associated data or the data will not be deposited. [Authors'comment: This review/perspective article contains no original data.]

Open Access This article is licensed under a Creative Commons Attribution 4.0 International License, which permits use, sharing, adaptation, distribution and reproduction in any medium or format, as long as you give appropriate credit to the original author(s) and the source, provide a link to the Creative Commons licence, and indicate if changes were made. The images or other third party material in this article are included in the article's Creative Commons licence, unless indicated otherwise in a credit line to the material. If material is not included in the article's Creative Commons licence and your intended use is not permitted by statutory regulation or exceeds the permitted use, you will need to obtain permission directly from the copyright holder. To view a copy of this licence, visit http://creativecomm ons.org/licenses/by/4.0/.

\section{References}

1. A.H. Zewail, Femtochemistry: atomic-scale dynamics of the chemical bond. J. Phys. Chem. A 104(24), 56605694 (2000)

2. P.B. Corkum, F. Krausz, Attosecond science. Nat. Phys. 3(6), 381-387 (2007)

3. F. Krausz, M. Ivanov, Attosecond physics. Rev. Mod. Phys. 81(1), 163-234 (2009)

4. M.J.J. Vrakking, Attosecond imaging. Phys. Chem. Chem. Phys. 16(7), 2775-2789 (2014)

5. C. Iaconis, I.A. Walmsley, Spectral phase interferometry for direct electric-field reconstruction of ultrashort optical pulses. Opt. Lett. 23(10), 792-794 (1998)

6. F. Lindner, M. Schätzel, H. Walther, A. Baltuška, E. Goulielmakis, F. Krausz, D. Milošević, D. Bauer, W. Becker, G. Paulus, Attosecond double-slit experiment. Phys. Rev. Lett. 95(4), 040401 (2005)

7. D. Shafir, H. Soifer, B.D. Bruner, M. Dagan, Y. Mairesse, S. Patchkovskii, M.Y. Ivanov, O. Smirnova,
N. Dudovich, Resolving the time when an electron exits a tunnelling barrier. Nature 485(7398), 343-346 (2012)

8. P.B. Corkum, Plasma perspective on strong field multiphoton ionization. Phys. Rev. Lett. 71, 1994 (1993)

9. P. Agostini, F. Fabre, G. Mainfray, G. Petite, N. Rahman, Free-free transitions following six-photon ionization of xenon atoms. Phys. Rev. Lett. 42(17), 11271130 (1979)

10. D. Bauer, P. Koval, Qprop: a Schrödinger-solver for intense laser-atom interaction. Comput. Phys. Commun. 174(5), 396-421 (2006)

11. K. Amini, J. Biegert, Chapter Three - Ultrafast electron diffraction imaging of gas-phase molecules, in volume 69 of Advances in Atomic, Molecular, and Optical Physics. (Academic Press, 2020), pp. 163-231

12. B. Bergues, Z. Ansari, D. Hanstorp, I.Y. Kiyan, Photodetachment in a strong laser field: an experimental test of Keldysh-like theories. Phys. Rev. A 75(6), 063415 (2007)

13. D.G. Arbó, K.L. Ishikawa, K. Schiessl, E. Persson, J. Burgdörfer, Intracycle and intercycle interferences in above-threshold ionization: the time grating. Phys. Rev. A 81, 021403(R) (2010)

14. M. Lein, J.P. Marangos, P.L. Knight, Electron diffraction in above-threshold ionization of molecules. Phys. Rev. A 66(5), 051404 (2002)

15. S.N. Yurchenko, S. Patchkovskii, I.V. Litvinyuk, P.B. Corkum, G.L. Yudin, Laser-induced interference, focusing, and diffraction of rescattering molecular photoelectrons. Phys. Rev. Lett. 93, 223003 (2004)

16. M. Spanner, O. Smirnova, P.B. Corkum, M.Y. Ivanov, Reading diffraction images in strong field ionization of diatomic molecules. J. Phys. B Atomic Mol. Opt. Phys. 37(12), 243-250 (2004)

17. Y. Huismans, A. Rouzée, A. Gijsbertsen, J.H. Jungmann, A.S. Smolkowska, P.S.W.M. Logman, F. Lépine, C. Cauchy, S. Zamith, T. Marchenko, J.M. Bakker, G. Berden, B. Redlich, A.F.G. van der Meer, H.G. Muller, W. Vermin, K.J. Schafer, M. Spanner, MYu. Ivanov, O. Smirnova, D. Bauer, S.V. Popruzhenko, M.J.J. Vrakking, Time-resolved holography with photoelectrons. Science 331(6013), 61-64 (2011)

18. M. Meckel, A. Staudte, S. Patchkovskii, D.M. Villeneuve, P.B. Corkum, R. Dörner, M. Spanner, Signatures of the continuum electron phase in molecular strong-field photoelectron holography. Nat. Phys. 10(8), 594-600 (2014)

19. S.G. Walt, N. Bhargava Ram, M. Atala, N.I. ShvetsovShilovski, A. von Conta, D. Baykusheva, M. Lein, H.J. Wörner, Dynamics of valence-shell electrons and nuclei probed by strong-field holography and rescattering. Nat. Commun. 8, 15651 (2017)

20. G. Porat, G. Alon, S. Rozen, O. Pedatzur, M. Krüger, D. Azoury, A. Natan, G. Orenstein, B.D. Bruner, M.J.J. Vrakking, N. Dudovich, Attosecond time-resolved photoelectron holography. Nat. Commun. 9(1), 2805 (2018)

21. M. Meckel, D. Comtois, D. Zeidler, A. Staudte, D. Pavicic, H.C. Bandulet, H. Pépin, J.C. Kieffer, R. Dörner, D.M. Villeneuve, P.B. Corkum, Laser-induced electron tunneling and diffraction. Science 320(5882), 1478-82 (2008) 
22. C.I. Blaga, J. Xu, A.D. Dichiara, E. Sistrunk, K. Zhang, P. Agostini, T.A. Miller, L.F. Dimauro, C.D. Lin, Imaging ultrafast molecular dynamics with laserinduced electron diffraction. Nature 483(7388), 194197 (2012)

23. B. Wolter, M.G. Pullen, A.T. Le, M. Baudisch, K. Doblhoff-Dier, A. Senftleben, M. Hemmer, C.D. Schröter, J. Ullrich, T. Pfeifer, R. Moshammer, S. Gräfe, O. Vendrell, C.D. Lin, J. Biegert, Ultrafast electron diffraction imaging of bond breaking in di-ionized acetylene. Science 354(6310), 308-312 (2016)

24. K. Amini, M. Sclafani, T. Steinle, A.-T. Le, A. Sanchez, C. Müller, J. Steinmetzer, L. Yue, J.R. Martinez Saavedra, M. Hemmer, M. Lewenstein, R. Moshammer, T. Pfeifer, M.G. Pullen, J. Ullrich, B. Wolter, R. Moszynski, F.J. de Abajo, C.D. Lin, S. Gräfe, J. Biegert, Imaging the renner-teller effect using laser-induced electron diffraction. Proc. Natl. Acad. Sci. 116(17), 8173-8177 (2019)

25. M. Kübel, Z. Dube, A.Y. Naumov, D.M. Villeneuve, P.B. Corkum, A. Staudte, Spatiotemporal imaging of valence electron motion. Nat. Commun. 10(1), 1 (2019)

26. G.S.J. Armstrong, M.A. Khokhlova, M. Labeye, A.S. Maxwell, E. Pisanty, M. Ruberti, Dialogue on analytical and ab initio methods in attoscience. Eur. Phys. J. D 75(7), 209 (2021)

27. W. Becker, F. Grasbon, R. Kopold, D.B. Milošević, G.G. Paulus, H. Walther, Above-threshold-ionization: from classical features to quantum effects. Adv. At. Mol. Opt. Phys. 48(1979), 35-98 (2002)

28. O. Jagutzki, A. Cerezo, A. Czasch, R. Dörner, M. Hattas, M. Huang, V. Mergel, U. Spillmann, K. Ullmann-Pfleger, T. Weber, H. Schmidt-Böcking, G.D.W. Smith, Multiple hit readout of a microchannel plate detector with a three-layer delay-line anode. IEEE Trans. Nucl. Sci. 49(5), 2477 (2002)

29. J. Ullrich, R. Moshammer, A. Dorn, R. Dörner, L.H. Schmidt, H. Schmidt-Böcking, Recoil-ion and electron momentum spectroscopy: reaction-microscopes. Rep. Prog. Phys. 66(9), 1463 (2003)

30. A.T.J.B. Eppink, D.H. Parker, Velocity map imaging of ions and electrons using electrostatic lenses: Application in photoelectron and photofragment ion imaging of molecular oxygen. Rev. Sci. Instrum. 68(9), 3477 (1997)

31. S.G. Eckart, Strong Field Ionization in Two-Color Fields (Goethe University Frankfurt, Frankfurt a. M. (Germany), 2019). (PhD Thesis)

32. A. Staudte, Subfemtosecond Electron Dynamics of $\mathrm{H}_{2}$ in Strong Fields (Goethe University Frankfurt, Frankfurt a. M. (Germany), 2005). (PhD Thesis)

33. P. Eckle, A.N. Pfeiffer, C. Cirelli, A. Staudte, R. Dörner, H.G. Muller, M. Büttiker, U. Keller, Attosecond ionization and tunneling delay time measurements in helium. Science 322(5907), 1525 (2008)

34. L. Torlina, F. Morales, J. Kaushal, I. Ivanov, A. Kheifets, A. Zielinski, A. Scrinzi, H.G. Muller, S. Sukiasyan, M. Ivanov, S. Olga, Interpreting attoclock measurements of tunnelling times. Nat. Phys. 11(6), 503 (2015)

35. N. Teeny, E. Yakaboylu, H. Bauke, C.H. Keitel, Ionization time and exit momentum in strong-field tunnel ionization. Phys. Rev. Lett. 116, 063003 (2016)
36. U.S. Sainadh, H. Xu, X. Wang, A. Atia-Tul-Noor, W.C. Wallace, N. Douguet, A. Bray, I. Ivanov, K. Bartschat, A. Kheifets, R.T. Sang, I.V. Litvinyuk, Attosecond angular streaking and tunnelling time in atomic hydrogen. Nature 568(7750), 75 (2019)

37. L.V. Keldysh, Ionization in the field of a strong electromagnetic wave. JETP 20, 1307-1314 (1965)

38. Cornelia Hofmann, Alexander Bray, Werner Koch, Hongcheng Ni, Nikolay I. Shvetsov-Shilovski, Quantum battles in attoscience: tunnelling. Eur. Phys. J. D 75(7), 208 (2021)

39. K. Liu, H. Ni, K. Renziehausen, J.-M. Rost, I. Barth, Deformation of atomic $p_{ \pm}$orbitals in strong elliptically polarized laser fields: Ionization time drifts and spatial photoelectron separation. Phys. Rev. Lett. 121, 203201 (2018)

40. H. Ni, U. Saalmann, J.-M. Rost, Tunneling exit characteristics from classical backpropagation of an ionized electron wave packet. Phys. Rev. A 97, 013426 (2018)

41. A.W. Bray, S. Eckart, A.S. Kheifets, KeldyshRutherford model for the attoclock. Phys. Rev. Lett. 121, 123201 (2018)

42. A.S. Alnaser, X.M. Tong, T. Osipov, S. Voss, C.M. Maharjan, B. Shan, Z. Chang, C.L. Cocke, Laser-peakintensity calibration using recoil-ion momentum imaging. Phys. Rev. A 70, 023413 (2004)

43. S. Eckart, M. Kunitski, M. Richter, A. Hartung, J. Rist, F. Trinter, K. Fehre, N. Schlott, K. Henrichs, L.H. Schmidt, T. Jahnke, M. Schöffler, K. Liu, I. Barth, J. Kaushal, F. Morales, M. Ivanov, O. Smirnova, R. Dörner, Ultrafast preparation and detection of ring currents in single atoms. Nat. Phys. 14, 701 (2018)

44. S. Eckart, K. Fehre, N. Eicke, A. Hartung, J. Rist, D. Trabert, N. Strenger, A. Pier, L.H. Schmidt, T. Jahnke, M.S. Schöffler, M. Lein, M. Kunitski, R. Dörner, Direct experimental access to the nonadiabatic initial momentum offset upon tunnel ionization. Phys. Rev. Lett. 121, 163202 (2018)

45. K. Liu, S. Luo, M. Li, Y. Li, Y. Feng, B. Du, Y. Zhou, P. Lu, I. Barth, Detecting and characterizing the nonadiabaticity of laser-induced quantum tunneling. Phys. Rev. Lett. 122, 053202 (2019)

46. R. Murray, W.-K. Liu, M.Y. Ivanov, Partial fouriertransform approach to tunnel ionization: atomic systems. Phys. Rev. A 81(2), 023413 (2010)

47. R. Murray, M. Spanner, S. Patchkovskii, M.Y. Ivanov, Tunnel ionization of molecules and orbital imaging. Phys. Rev. Lett. 106(17), 173001 (2011)

48. D. Comtois, H.-C. Bandulet, M. Spanner, D. Pavičić, M. Meckel, D. Zeidler, H. Pépin, R. Dörner, J.-C. Kieffer, D.M. Villeneuve, P.B. Corkum, A. Staudte, Laserinduced orbital projection and diffraction of $\mathrm{O}_{-2}$ with velocity map imaging. J. Mod. Opt. 60(17), 1395-1408 (2013)

49. H. Akagi, T. Otobe, A. Staudte, A. Shiner, F. Turner, R. Dörnerr, D.M. Villeneuve, P.B. Corkum, Laser tunnel ionization from multiple orbitals in $\mathrm{HCl}$. Science 325, 1364 (2009)

50. A. Staudte, S. Patchkovskii, D. Pavičić, H. Akagi, O. Smirnova, D. Zeidler, M. Meckel, D.M. Villeneuve, R. Dörner, M.Y. Ivanov, P.B. Corkum, Angular tunneling ionization probability of fixed-in-space $\mathrm{H}_{2}$ molecules in 
intense laser pulses. Phys. Rev. Lett. 102(3), 033004 (2009)

51. L. Holmegaard, J.L. Hansen, L. Kalhøj, S. Louise Kragh, H. Stapelfeldt, F. Filsinger, J. Küpper, G. Meijer, D. Dimitrovski, M. Abu-Samha, C.P.J. Martiny, L. Bojer Madsen, Photoelectron angular distributions from strong-field ionization of oriented molecules. Nat. Phys. 6(6), 428-432 (2010)

52. A. Fleischer, H.J. Wörner, L. Arissian, L.R. Liu, M. Meckel, A. Rippert, R. Dörner, D.M. Villeneuve, P.B. Corkum, A. Staudte, Probing angular correlations in sequential double ionization. Phys. Rev. Lett. 107, 113003 (2011)

53. D. Akoury, K. Kreidi, T. Jahnke, T. Weber, A. Staudte, M. Schöffler, N. Neumann, J. Titze, LPh.H. Schmidt, A. Czasch, O. Jagutzki, R.A. Costa Fraga, R.E. Grisenti, R. Díez Muiño, N.A. Cherepkov, S.K. Semenov, P. Ranitovic, C.L. Cocke, T. Osipov, H. Adaniya, J.C. Thompson, M.H. Prior, A. Belkacem, A.L. Landers, H. Schmidt-Böcking, R. Dörner, The simplest double slit: Interference and entanglement in double photoionization of $\mathrm{H}_{2}$. Science 318(5852), 949 (2007)

54. M. Kunitski, N. Eicke, P. Huber, J. Köhler, S. Zeller, J. Voigtsberger, N. Schlott, K. Henrichs, H. Sann, F. Trinter, L.H. Schmidt, A. Kalinin, M.S. Schöffler, T. Jahnke, M. Lein, R. Dörner, Double-slit photoelectron interference in strong-field ionization of the neon dimer. Nat. Commun. 10(1), 1 (2019)

55. S. Eckart, M. Kunitski, I. Ivanov, M. Richter, K. Fehre, A. Hartung, J. Rist, K. Henrichs, D. Trabert, N. Schlott, L.H. Schmidt, T. Jahnke, M.S. Schöffler, A. Kheifets, R. Dörner, Subcycle interference upon tunnel ionization by counter-rotating two-color fields. Phys. Rev. A 97, 041402(R) (2018)

56. S. Eckart, D. Trabert, K. Fehre, A. Geyer, J. Rist, K. Lin, F. Trinter, L.H. Schmidt, M.S. Schöffler, T. Jahnke, M. Kunitski, R. Dörner, Sideband modulation by subcycle interference. Phys. Rev. A 102, 043115 (2020)

57. M. Han, P. Ge, Y. Shao, Q. Gong, Y. Liu, Attoclock photoelectron interferometry with two-color corotating circular fields to probe the phase and the amplitude of emitting wave packets. Phys. Rev. Lett. 120, 073202 (2018)

58. P. Ge, M. Han, Y. Deng, Q. Gong, Y. Liu, Universal description of the attoclock with two-color corotating circular fields. Phys. Rev. Lett. 122, 013201 (2019)

59. S. Eckart, Holographic angular streaking of electrons and the wigner time delay. Phys. Rev. Res. 2, 033248 (2020)

60. D. Trabert, K. Fehre, N. Anders, A. Geyer, S. Grundmann, M. Schöffler, L.H. Schmidt, T. Jahnke, R. Dörner, M. Kunitski, S. Eckart, Angular dependence of the wigner time delay upon tunnel ionization of $\mathrm{H}_{2}$. Nat. Commun. 12, 1697 (2021)

61. P. Eugene, Wigner, Lower limit for the energy derivative of the scattering phase shift. Phys. Rev. 98, 145 (1955)

62. J. Vos, L. Cattaneo, S. Patchkovskii, T. Zimmermann, C. Cirelli, M. Lucchini, A. Kheifets, A.S. Landsman, U. Keller, Orientation-dependent stereo Wigner time delay and electron localization in a small molecule. Science 360(6395), 1326 (2018)

63. Y. Feng, M. Li, S. Luo, K. Liu, B. Du, Y. Zhou, P. Lu, Semiclassical analysis of photoelectron interference in a synthesized two-color laser pulse. Phys. Rev. A 100, 063411 (2019)

64. Lucas J. Zipp, Adi Natan, Philip H. Bucksbaum, Probing electron delays in above-threshold ionization. Optica 1(6), 361-364 (2014)

65. S. Kerbstadt, D. Pengel, D. Johannmeyer, L. Englert, T. Bayer, M. Wollenhaupt, Control of photoelectron momentum distributions by bichromatic polarizationshaped laser fields. New J. Phys. 19(10), 103017 (2017)

66. T. Remetter, P. Johnsson, J. Mauritsson, K. Varjú, F. Ni, Y. Lépine, E. Gustafsson, M. Kling, J. Khan, K.J. López-Martens, R. Schafer, M.J.J. Vrakking, A. L'Huillier, Attosecond electron wave packet interferometry. Nat. Phys. 30(2), 323-326 (2006)

67. A. Chacon, M. Lein, C. Ruiz, Retrieval of the amplitude and phase of the dipole matrix element by attosecond electron-wave-packet interferometry. Phys. Rev. A 87, 023408 (2013)

68. J.J. Sakurai, Jim Napolitano, Modern Quantum Mechanics, 2nd edn. (Cambridge University Press, Cambridge, 2017)

69. B. Yang, K.J. Schafer, B. Walker, K.C. Kulander, P. Agostini, L.F. DiMauro, Intensity-dependent scattering rings in high order above-threshold ionization. Phys. Rev. Lett. 71, 3770-3773 (1993)

70. S. Skruszewicz, J. Tiggesbäumker, K.H. Meiwes-Broer, M. Arbeiter, Th. Fennel, D. Bauer, Two-color strongfield photoelectron spectroscopy and the phase of the phase. Phys. Rev. Lett. 115(4), 1-6 (2015)

71. M. Haertelt, X.-B. Bian, M. Spanner, A. Staudte, P.B. Corkum, Probing molecular dynamics by laser-induced backscattering holography. Phys. Rev. Lett. 116(13), 133001 (2016)

72. H. Kang, A.S. Maxwell, D. Trabert, X. Lai, S. Eckart, M. Kunitski, M. Schöffler, T. Jahnke, X. Bian, R. Dörner, C. F. de M. Faria, Holographic detection of parity in atomic and molecular orbitals. Phys. Rev. A 102, 013109 (2020)

73. C. F. de M Faria, A. S. Maxwell, It is all about phases: ultrafast holographic photoelectron imaging. Reports on Progress in Physics 83(3), 034401 (2020)

74. T. Zuo, A.D. Bandrauk, P.B. Corkum, Laser-induced electron diffraction: a new tool for probing ultrafast molecular dynamics. Chem. Phys. Lett. 259(3-4), 313320 (1996)

75. C.D. Lin, A.-T. Le, Z. Chen, T. Morishita, R. Lucchese, Strong-field rescattering physics-self-imaging of a molecule by its own electrons. J. Phys. B At. Mol. Opt. Phys. 43(12), 122001 (2010)

76. M. Okunishi, H. Niikura, R.R. Lucchese, T. Morishita, K. Ueda, Extracting electron-ion differential scattering cross sections for partially aligned molecules by laserinduced rescattering photoelectron spectroscopy. Phys. Rev. Lett 106(6), 063001 (2011)

77. J. Xu, C.I. Blaga, A.D. DiChiara, E. Sistrunk, K. Zhang, Z. Chen, A.-T. Le, T. Morishita, C.D. Lin, P. Agostini, L.F. DiMauro, Laser-induced electron diffraction for probing rare gas atoms. Phys. Rev. Lett 109(23), 233002 (2012) 
78. J. Xu, C.I. Blaga, K. Zhang, Y.H. Lai, C.D. Lin, T.A. Miller, P. Agostini, L.F. DiMauro, Diffraction using laser-driven broadband electron wave packets. Nat. Commun. 5(1), 1-6 (2014)

79. B. Wolter, M.G. Pullen, M. Baudisch, M. Sclafani, M. Hemmer, A. Senftleben, C.D. Schröter, J. Ullrich, R. Moshammer, J. Biegert, Strong-field physics with midIR fields. Phys. Rev. X 5(2), 021034 (2015)

80. M.G. Pullen, B. Wolter, A.-T. Le, M. Baudisch, M. Hemmer, A. Senftleben, C.D. Schröter, J. Ullrich, R. Moshammer, C.-D. Lin, J. Biegert, Imaging an aligned polyatomic molecule with laser-induced electron diffraction. Nat. Commun. 6(1), 1-6 (2015)

81. M.G. Pullen, B. Wolter, A.-T. Le, M. Baudisch, M. Sclafani, H. Pires, C.D. Schroeter, J. Ullrich, R. Moshammer, T. Pfeifer, C.D. Lin, J. Biegert, Influence of orbital symmetry on diffraction imaging with rescattering electron wave packets. Nat. Commun. 7(1), 1-6 (2016)

82. K. Amini, M. Sclafani, T. Steinle, A.-T. Le, A. Sanchez, C. Müller, J. Steinmetzer, L. Yue, J.R.M. Saavedra, M. Hemmer et al., Imaging the renner-teller effect using laser-induced electron diffraction. Proc. Natl. Acad. Sci. 116(17), 8173-8177 (2019)

83. K. Amini, J. Biegert, F. Calegari, A. Chacón, M.F. Ciappina, A. Dauphin, D.K. Efimov, C. F. de M Faria, Symphony on strong field approximation. Reports on Progress in Physics 82(11), 116001 (2019)

84. K. Ueda, E. Sokell, S. Schippers, F. Aumayr, H. Sadeghpour, J. Burgdörfer, C. Lemell, X.-M. Tong, T. Pfeifer, F. Calegari, A. Palacios, F. Martín, P. Corkum, G. Sansone, E. Gryzlova, A. GrumGrzhimailo, M. Piancastelli, P. Weber, T. Steinle, K. Tanaka, Roadmap on photonic, electronic and atomic collision physics: I. light-matter interaction. J. Phys. B At. Mol. Opt. Phys. 52(17), 171001 (2019)

85. H. Fuest, Y.H. Lai, C.I. Blaga, K. Suzuki, J. Xu, P. Rupp, H. Li, P. Wnuk, P. Agostini, K. Yamazaki, M. Kanno, H. Kono, M.F. Kling, L.F. DiMauro, Diffractive imaging of $\mathrm{C}_{60}$ structural deformations induced by intense femtosecond midinfrared laser fields. Phys. Rev. Lett 122(5), 053002 (2019)

86. X. Liu, K. Amini, T. Steinle, A. Sanchez, M. Shaikh, B. Belsa, J. Steinmetzer, A.-T. Le, R. Moshammer, T. Pfeifer et al., Imaging an isolated water molecule using a single electron wave packet. J. Chem. Phys. 151(2), 024306 (2019)

87. B. Belsa, K. Amini, X. Liu, A. Sanchez, T. Steinle, J. Steinmetzer, A.-T. Le, R. Moshammer, T. Pfeifer et al., Laser-induced electron diffraction of the ultrafast umbrella motion in ammonia. Under review (2020)

88. A. Sanchez, K. Amini, S.-J. Wang, T. Steinle, B. Belsa, A.-T. Le, X. Liu, R. Moshammer, T. Pfeifer et al., Molecular structure retrieval directly from laboratoryframe photoelectron spectra in laser-induced electron diffraction. Under review (2020)

89. X. Liu, K. Amini, A. Sanchez, B. Belsa, T. Steinle, A.-T. Le, R. Moshammer, T. Pfeifer et al., Machine learning for laser-induced electron diffraction. Under review (2021)

90. K.C. Kulander, K.J. Schafer, J.L. Krause, Dynamics of short-pulse excitation, ionization and harmonic conver- sion, in Super-Intense Laser-Atom Physics. (Springer, 1993), pp. 95-110

91. K.J. Schafer, B. Yang, L.F. DiMauro, K.C. Kulander, Above threshold ionization beyond the high harmonic cutoff. Phys. Rev. Lett 70(11), 1599 (1993)

92. S. Varró, F. Ehlotzky, A new integral equation for treating high-intensity multiphoton processes. Il Nuovo Cimento D 15(11), 1371-1396 (1993)

93. L. Schäfer, Electron diffraction as a tool of structural chemistry. Appl. Spectroscopy 30(2), 123-149 (1976)

94. P.D. McCaffrey, J.K. Dewhurst, D.W.H. Rankin, R.J. Mawhorter, S. Sharma, Interatomic contributions to high-energy electron-molecule scattering. J. Chem. Phys. 128(20), 204304 (2008)

95. M. Hargittai et al., Stereochemical Applications of GasPhase Electron Diffraction, Part A, vol. 4 (John Wiley \& Sons, New York, 1988)

96. R. Moshammer, M. Unverzagt, W. Schmitt, J. Ullrich, H. Schmidt-Böcking, A $4 \pi$ recoil-ion electron momentum analyzer: a high-resolution microscope for the investigation of the dynamics of atomic, molecular and nuclear reactions. Nucl. Instrum. Methods Phys. Res. Sect. B Beam Interact. Mater. Atoms 108(4), 425-445 (1996)

97. R. Dörner, V. Mergel, O. Jagutzki, L. Spielberger, J. Ullrich, R. Moshammer, H. Schmidt-Böcking, Cold target recoil ion momentum spectroscopy: a momentum microscope to view atomic collision dynamics. Phys. Rep. 330(2-3), 95-192 (2000)

98. Z. Chen, A.-T. Le, T. Morishita, C.D. Lin, Quantitative rescattering theory for laser-induced high-energy plateau photoelectron spectra. Phys. Rev. A 79(3), 033409 (2009)

99. J. Yang, X. Zhu, T.J.A. Wolf, Z. Li, J.P.F. Nunes, R. Coffee, J.P. Cryan, M. Gühr, K. Hegazy, T.F. Heinz et al., Imaging $\mathrm{CF}_{3} \mathrm{I}$ conical intersection and photodissociation dynamics with ultrafast electron diffraction. Science 361(6397), 64-67 (2018)

100. T.J.A. Wolf, D.M. Sanchez, J. Yang, R.M. Parrish, J.P.F. Nunes, M. Centurion, R. Coffee, J.P. Cryan, M. Gühr, K. Hegazy et al., The photochemical ringopening of 1, 3-cyclohexadiene imaged by ultrafast electron diffraction. Nat. Chem. 11(6), 504-509 (2019)

101. J. Yang, X. Zhu, J.P.F. Nunes, K.Y. Jimmy, R.M. Parrish, T.J.A. Wolf, M. Centurion, M. Gühr, R. Li, Y. Liu et al., Simultaneous observation of nuclear and electronic dynamics by ultrafast electron diffraction. Science 368(6493), 885-889 (2020)

102. D.B. Milošević, G.G. Paulus, J. Phys, W. Becker, J. Phys. B: At. Mol. Opt. Phys. 39, R203 (2006)

103. R. Kopold, W. Becker, M. Kleber, Opt. Commun. 179, $39(2000)$

104. R. Kopold, D.B. Milošević, W. Becker, Phys. Rev. Lett. 84, $3831(2000)$

105. P. Salières, B. Carré, L. Le Déroff, F. Grasbon, G.G. Paulus, H. Walther, R. Kopold, W. Becker, D.B. Milošević, A. Sanpera, M. Lewenstein, Science 292, 902 (2001)

106. D.B. Milošević, D. Bauer, W. Becker, J. Mod. Opt. 53, 125 (2006)

107. S.V. Popruzhenko, D. Bauer, J. Mod. Opt. 55, 2573 (2008) 
108. T.M. Yan, S.V. Popruzhenko, D. Bauer, Trajectorybased coulomb-corrected strong field approximation, in Progress in Ultrafast Intense Laser Science. ed. by K. Yamanouchi, K. Midorikawa (Springer-Verlag, Heidelberg, 2013), pp. 1-16

109. X.-Y. Lai, C. Poli, H. Schomerus, C. Figueira, de Morisson Faria. Phys. Rev. A 92, 043407 (2015)

110. X.Y. Lai, S.G. Yu, Y.Y. Huang, L.Q. Hua, C. Gong, W. Quan, C. Figueira, de Morisson Faria. J. Liu. Phys. Rev. A 96, 013414 (2017)

111. A.S. Maxwell, A. Al-Jawahiry, T. Das, C. Figueira, de Morisson Faria. Phys. Rev. A 96, 023420 (2017)

112. D. Dimitrovski, L.B. Madsen, Phys. Rev. A 91, 033409 (2015)

113. N.I. Shvetsov-Shilovski, M. Lein, L.B. Madsen, E. Räsänen, C. Lemell, J. Burgdörfer, D.G. Arbó, K. Tőkési, Phys. Rev. A 94, 013415 (2016)

114. N.I. Shvetsov-Shilovski, M. Lein, Phys. Rev. A 97, 013411 (2018)

115. B. Hu, J. Liu, S.G. Chen, Phys. Lett. A 236, 533 (1997)

116. C. Hofmann, A.S. Landsman, C. Cirelli, A.N. Pfeiffer, U. Keller, J. Phys. B At. Mol. Opt. Phys. 46, 125601 (2013)

117. B. Wolter, C. Lemell, M. Baudisch, M.G. Pullen, X.M. Tong, M. Hemmer, A. Senftleben, C.D. Schröter, J. Ullrich, R. Moshammer, J. Biegert, J. Burgdörfer. Phys. Rev. A 90, 063424 (2014)

118. M. Li, J.-W. Geng, H. Liu, Y. Deng, C. Wu, L.-Y. Peng, Q. Gong, Y. Liu, Phys. Rev. Lett. 112, 113002 (2014)

119. C.I. Blaga, F. Catoire, P. Colosimo, G.G. Paulus, H.G. Muller, P. Agostini, L.F. DiMauro, Nat. Phys. 5, 335 (2009)

120. X.-B. Bian, Y. Huismans, O. Smirnova, K.-J. Yuan, M.J.J. Vrakking, A.D. Bandrauk, Phys. Rev. A 84, 043420 (2011)

121. D.D. Hickstein, P. Ranitovic, S. Witte, X.-M. Tong, Y. Huismans, P. Arpin, X. Zhou, K.E. Keister, C.W. Hogle, B. Zhang, C. Ding, P. Johnsson, N. Toshima, M.J.J. Vrakking, M.M. Murnane, H.C. Kapteyn, Phys. Rev. Lett. 109, 073004 (2012)

122. M. Kübel, M. Arbeiter, C. Burger, N.G. Kling, T. Pischke, R. Moshammer, T. Fennel, M.F. Kling, B. Bergues, J. Phys. B At. Mol. Opt. Phys. 51, 134007 (2018)

123. M. Kübel, P. Wustelt, Y. Zhang, S. Skruszewicz, D. Hoff, D. Würzler, H. Kang, D. Zille, D. Adolph, G.G. Paulus, A.M. Sayler, M. Dumergue, A. Nayak, R. Flender, L. Haizer, M. Kurucz, B. Kiss, S. Kühn, B. Fetić, D.B. Milošević, High-order phase-dependent asymmetry in the above-threshold ionization plateau. Phys. Rev. Lett. 126, 113201 (2021)

124. D. Bauer, D.B. Milošević, W. Becker, On the validity of the strong field approximation and simple man is theory. J. Mod. Opt. 53, 135 (2006)

125. J.-W. Geng, L. Qin, M. Li, W.-H. Xiong, Y. Liu, Q. Gong, L.-Y. Peng, J. Phys. B At. Mol. Opt. Phys. 47, 204027 (2014)

126. D.G. Arbó, C. Lemell, S. Nagele, N. Camus, L. Fechner, A. Krupp, T. Pfeifer, S.D. López, R. Moshammer, J. Burgdörfer. Phys. Rev. A 92, 023402 (2015)

127. A.S. Maxwell, C. Figueira, de Morisson Faria. J. Phys. B At. Mol. Opt. Phys. 51, 124001 (2018)
128. B. Fetić, W. Becker, D.B. Milošević, Phys. Rev. A 102, 023101 (2020)

129. P. Roman, Advanced Quantum Theory: an Outline of the Fundamental Ideas (Addison-Wesley, Reading, 1965)

130. A.F. Starace, Handbuch Der Physik, vol. 31 (Springer, Berlin, 1982)

131. L.A.A. Nikolopoulos, T.K. Kjeldsen, L.B. Madsen, Phys. Rev. A 75, 063426 (2007)

132. P.A. Golovinsky, I. Yu Kiyan, V.S. Rostovtsev, J. Phys. B 23, 2743 (1990)

133. Nina Rohringer, Robin Santra, Multichannel coherence in strong-field ionization. Phys. Rev. A 79(5), 53402 (2009)

134. E. Goulielmakis, Z.-H. Loh, A. Wirth, R. Santra, N. Rohringer, V.S. Yakovlev, S. Zherebtsov, T. Pfeifer, A.M. Azzeer, M.F. Kling, S.R. Leone, F. Krausz, Realtime observation of valence electron motion. Nature 466(7307), 739-743 (2010)

135. F. Calegari, D. Ayuso, A. Trabattoni, L. Belshaw, S. De Camillis, S. Anumula, F. Frassetto, L. Poletto, A. Palacios, P. Decleva, J.B. Greenwood, F. Martín, M. Nisoli, Ultrafast electron dynamics in phenylalanine initiated by attosecond pulses. Science 346(6207), 336339 (2014)

136. P.M. Kraus, B. Mignolet, D. Baykusheva, A. Rupenyan, L. Hornỳ, E.F. Penka, G. Grassi, O.I. Tolstikhin, J. Schneider, F. Jensen, L.B. Madsen, A.D. Bandrauk, F. Remacle, H.J. Wörner, Measurement and laser control of attosecond charge migration in ionized iodoacetylene. Science 350(6262), 790-795 (2015)

137. T. Ergler, A. Rudenko, B. Feuerstein, K. Zrost, C. Schröter, R. Moshammer, J. Ullrich, Spatiotemporal imaging of ultrafast molecular motion: collapse and revival of the $\mathrm{D}_{2}^{+}$nuclear wave packet. Phys. Rev. Lett. 97(19), 193001 (2006)

138. F. Rosca-Pruna, M.J.J. Vrakking, Experimental observation of revival structures in picosecond laser-induced alignment of $\mathrm{I}_{2}$. Phys. Rev. Lett. 87(15), 153902 (2001)

139. H. Stapelfeldt, T. Seideman, Colloquium: aligning molecules with strong laser pulses. Rev. Mod. Phys. 75(2), 543-557 (2003)

140. E.T. Karamatskos, S. Raabe, T. Mullins, A. Trabattoni, P. Stammer, G. Goldsztejn, R.R. Johansen, K. Długołecki, H. Stapelfeldt, M.J.J. Vrakking, S. Trippel, A. Rouzée, J. Küpper, Molecular movie of ultrafast coherent rotational dynamics of OCS. Nat. Commun. 10(1), 1-7 (2019)

141. J. Wu, M. Kunitski, M. Pitzer, F. Trinter, L.H. Schmidt, T. Jahnke, M. Magrakvelidze, C.B. Madsen, L.B. Madsen, U. Thumm, R. Dörner, Electron-nuclear energy sharing in above-threshold multiphoton dissociative ionization of $\mathrm{H}_{2}$. Phys. Rev. Lett. 111(2), 23002 (2013)

142. L. Hackermüller, K. Hornberger, B. Brezger, A. Zeilinger, M. Arndt, Decoherence of matter waves by thermal emission of radiation. Nature 427(6976), 711 (2004)

143. Andrei Benediktovitch, Vinay P. Majety, Nina Rohringer, Quantum theory of superfluorescence based on two-point correlation functions. Phys. Rev. A 99, 013839 (2019) 
144. A.A. Milner, A. Korobenko, J.W. Hepburn, V. Milner, Effects of ultrafast molecular rotation on collisional decoherence. Phys. Rev. Lett. 113, 043005 (2014)

145. B.A. Stickler, F.T. Ghahramani, K. Hornberger, Rotational alignment decay and decoherence of molecular superrotors. Phys. Rev. Lett. 121, 243402 (2018)

146. P. Mohanty, E.M.Q. Jariwala, R.A. Webb, Intrinsic decoherence in mesoscopic systems. Phys. Rev. Lett. 78, 3366-3369 (1997)

147. M. Lewenstein, P. Balcou, M.Y. Ivanov, A. L'Huillier, P.B. Corkum, Theory of high-harmonic generation by low-frequency laser fields. Phys. Rev. A 49, 2117 (1994)

148. J. Heimerl, T. Higuchi, M. Ammon, M.A. Schneider, P. Hommelhoff, Gap-size dependence of optical near fields in a variable nanoscale two-tip junction. Phys. Rev. B 101, 125403 (2020)

149. S. Ghimire, D.A. Reis, High-harmonic generation from solids. Nat. Phys. 15(1), 10-16 (2019)

150. S. Ghimire, A.D. DiChiara, E. Sistrunk, P. Agostini, L.F. DiMauro, D.A. Reis, Observation of high-order harmonic generation in a bulk crystal. Nat. Phys. 7(2), 138-141 (2011)

151. H. Liu, Y. Li, Y.S. You, S. Ghimire, T.F. Heinz, D.A. Reis, High-harmonic generation from an atomically thin semiconductor. Nat. Phys. 13(3), 262 (2017)

152. G. Vampa, C.R. McDonald, G. Orlando, D.D. Klug, P.B. Corkum, T. Brabec, Theoretical analysis of highharmonic generation in solids. Phys. Rev. Lett. 113(7), 073901 (2014)

153. G. Vampa, T.J. Hammond, N. Thiré, B.E. Schmidt, F. Légaré, C.R. McDonald, T. Brabec, P.B. Corkum, Linking high harmonics from gases and solids. Nature 522(7557), 462 (2015)

154. G. Vampa, T. Brabec, Merge of high harmonic generation from gases and solids and its implications for attosecond science. J. Phys. B At. Mol. Opt. Phys. 50(8), 083001 (2017)

155. E.N. Osika, A. Chacón, L. Ortmann, N. Suárez, J.A. Pérez-Hernández, B. Szafran, M.F. Ciappina, F. Sols, A.S. Landsman, M. Lewenstein, Wannier-Bloch approach to localization in high-harmonics generation in solids. Phys. Rev. X 7(2), 021017 (2017)

156. X.-L. Qi, S.-C. Zhang, Topological insulators and superconductors. Rev. Mod. Phys. 83(4), 1057-1110 (2011)

157. R.B. Laughlin, Nobel lecture: fractional quantization. Rev. Mod. Phys. 71(4), 863-874 (1999)

158. F. Duncan, M. Haldane, Nobel lecture: topological quantum matter. Rev. Mod. Phys. 89(4), 040502 (2017)

159. Y. Murakami, M. Eckstein, P. Werner, High-harmonic generation in mott insulators. Phys. Rev. Lett. 121(5), 57405 (2018)

160. R.E.F. Silva, I.V. Blinov, A.N. Rubtsov, O. Smirnova, M. Ivanov, High-harmonic spectroscopy of ultrafast many-body dynamics in strongly correlated systems. Nat. Photon. 12(2), 266-270 (2018)

161. T. Okugawa, P. Tang, A. Rubio, D.M. Kennes, Topological phase transitions induced by disorder in magnetically doped $(\mathrm{Bi}, \mathrm{Sb})_{2} \mathrm{Te}_{3}$ thin films. Phys. Rev. B 102, 201405 (2020)
162. M. Lysne, Y. Murakami, M. Schüler, P. Werner, Highharmonic generation in spin-orbit coupled systems. Phys. Rev. B 102, 081121 (2020)

163. D. Baykusheva, A. Chacón, D. Kim, D.E. Kim, D.A. Reis, S. Ghimire. Strong-field physics in threedimensional topological insulators, (2020)

164. S. Takayoshi, Y. Murakami, P. Werner, High-harmonic generation in quantum spin systems. Phys. Rev. B 99(18), 184303 (2019)

165. M. Borsch, C.P. Schmid, L. Weigl, S. Schlauderer, N. Hofmann, C. Lange, J.T. Steiner, S.W. Koch, R. Huber, M. Kira, Super-resolution lightwave tomography of electronic bands in quantum materials. Science 370(6521), 1204-1207 (2020)

166. A. Chacón, D. Kim, W. Zhu, S.P. Kelly, A. Dauphin, E. Pisanty, A.S. Maxwell, A. Picón, M.F. Ciappina, D.E. Kim, C. Ticknor, A. Saxena, M. Lewenstein, Circular dichroism in higher-order harmonic generation: heralding topological phases and transitions in chern insulators. Phys. Rev. B 102, 134115 (2020)

167. F. Langer, C.P. Schmid, S. Schlauderer, M. Gmitra, J. Fabian, P. Nagler, C. Schüller, T. Korn, P.G. Hawkins, J.T. Steiner, U. Huttner, S.W. Koch, M. Kira, R. Huber, Lightwave valleytronics in a monolayer of tungsten diselenide. Nature 557(77), 76-80 (2018)

168. B. Buades, I. Leon, N. Di Palo, D. Rivas, T.P.H. Sidiropolous, S. Severino, M. Reduzzi, S. Cousin, M. Hemmer, E. Caterina Cocchi, J. Herrero. Pellegrin, S.. Ma. Martin, nas Valero, E. Coronado, T. Danz, C. Draxl, M. Uemoto, K. Yabana, M. Schultze, S. Wall, A. Picon, J. Biegert. Attosecond soft-X-ray spectroscopy of a transition metal dichalcogenide material. In Nonlinear Optics (NLO), page NF1A.5. Optical Society of America, (2019)

169. R.E.F. Silva, Á. Jiménez-Galán, B. Amorim, O. Smirnova, M. Ivanov, Topological strong-field physics on sub-laser-cycle timescale. Nat. Photon. 13(12), 849854 (2019)

170. D. Bauer, K.K. Hansen, High-harmonic generation in solids with and without topological edge states. Phys. Rev. Lett. 120(6), 177401 (2018)

171. H. Zhang, C.-X. Liu, X.-L. Qi, X. Dai, Z. Fang, S.-C. Zhang, Topological insulators in $\mathrm{Bi}_{2} \mathrm{Se}_{3}, \mathrm{Bi}_{2} \mathrm{Te}_{3}$ and $\mathrm{Sb}_{2} \mathrm{Te}_{3}$ with a single Dirac cone on the surface. Nat. Phys. 5(6), 438-442 (2009)

172. Y.L. Chen, J.G. Analytis, J.-H. Chu, Z.K. Liu, S.-K. Mo, X.L. Qi, H.J. Zhang, D.H. Lu, X. Dai, Z. Fang, S.C. Zhang, I.R. Fisher, Z. Hussain, Z.-X. Shen, Experimental realization of a three-dimensional topological insulator, $\mathrm{Bi}_{2} \mathrm{Te}_{3}$. Science 325(5937), 178-181 (2009)

173. X. Lu, P. Stepanov, W. Yang, M. Xie, M.A. Aamir, I. Das, C. Urgell, K. Watanabe, T. Taniguchi, G. Zhang, A. Bachtold, A.H. MacDonald, D.K. Efetov, Superconductors, orbital magnets and correlated states in magic-angle bilayer graphene. Nature 574, 653-657 (2019)

174. M. Neupane, A. Richardella, J. Sánchez-Barriga, S.Y. $\mathrm{Xu}$, N. Alidoust, I. Belopolski, C. Liu, G. Bian, D. Zhang, D. Marchenko, A. Varykhalov, O. Rader, M. Leandersson, T. Balasubramanian, T. Chang, H. Jeng, S. Basak, H. Lin, A. Bansil, N. Samarth, M.Z. Hasan, Observation of quantum-tunnelling-modulated 
spin texture in ultrathin topological insulator $\mathrm{Bi}_{2} \mathrm{Se}_{3}$ films. Nat. Commun. 5(3841), 1-7 (2014)

175. F.D.M. Haldane, Model for a quantum Hall effect without Landau levels: condensed-matter realization of the parity anomaly. Phys. Rev. Lett. 61(18), 2015-2018 (1988)

176. N.P. Armitage, E.J. Mele, Ashvin Vishwanath, Weyl and Dirac semimetals in three-dimensional solids. Rev. Mod. Phys. 90, 015001 (2018)

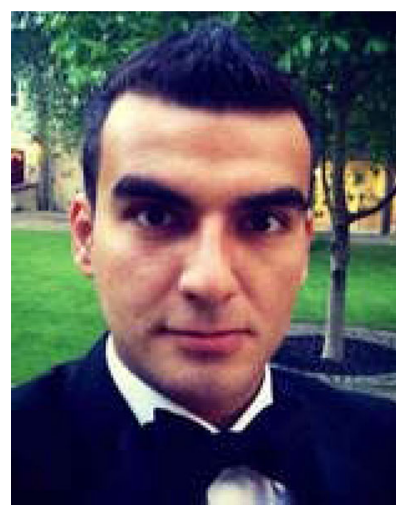

Kasra Amini completed his D.Phil in Physical and Theoretical Chemistry at the University of Oxford in 2017, investigating ultrafast molecular photofragmentation and dynamics with femtosecond Coulomb explosion imaging (CEI). Prior to this, he completed a joint-honors integrated undergraduatemaster degree in Chemistry with Mathematics at University College London. He has held postdoctoral and research fellow positions at ICFO, Barcelona, under the supervision of Prof. Dr. Jens Biegert, studying laser-induced electron diffraction and CEI of gasphase molecules. He is currently a junior research group leader at the Max-Born-Institute, Berlin, leading a group in ultrafast electron diffraction.

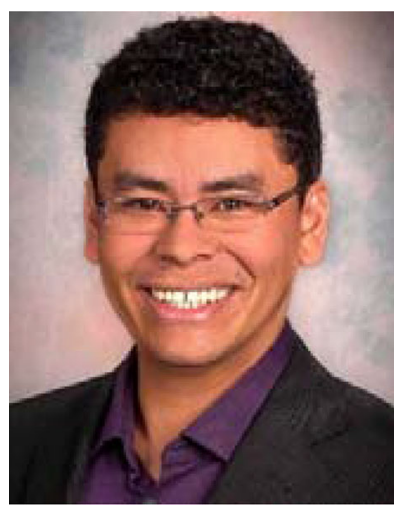

Alexis Chacón obtained his $\mathrm{PhD}$ from Universidad de Salamanca under the supervision of Prof. C. Ruiz and Prof. L. Plaja. He specialized in the physics of electron wavepacket characterization via attosecond quantum interferometry. His first postdoc was done in the Quantum Theory Group of Prof. M. Lewenstein. Afterward, he joined the LANL to investigate topological phases and transitions of matter using nonlinear optical responses. He joined the SLAC-SU theoretical-experimental collaboration and contributed to the realization of $3 \mathrm{D}$ topological insulators at surface states. Since October 2019, he is Group Leader at MPI-Korea

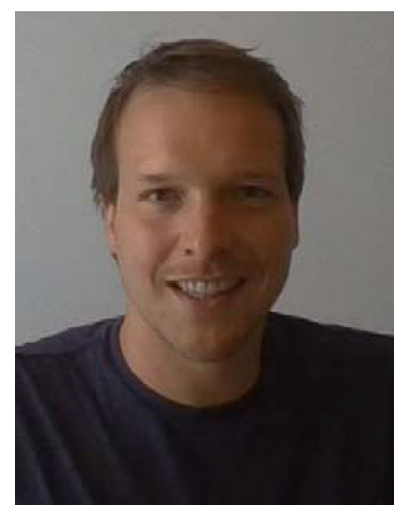

Sebastian Eckart has studied Physics at the University of Konstanz (Germany). During his master thesis, he worked on the ultra-sensitive detection of $\mathrm{THz}$ fields. Since 2015, he is working at the University of Frankfurt (Germany) in the group of Prof. Reinhard Dörner and experimentally and theoretically investigates strong field ionization of single atoms and small molecules. His research interests include non-sequential double ionization, sub-cycle interference, and non-adiabatic tunneling. He finished his $\mathrm{PhD}$ in 2019 and currently works as a postdoc on a better understanding of holographic angular streaking of electrons (HASE).

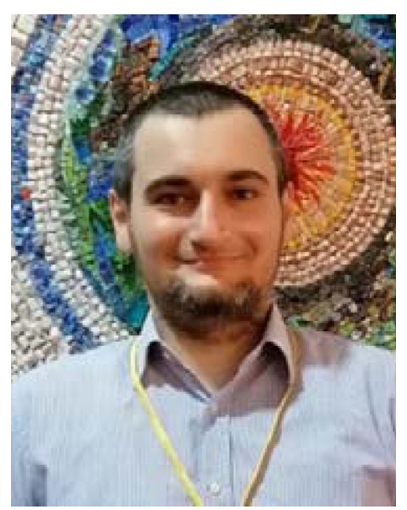

Benjamin Fetić completed his bachelor, master, and $\mathrm{PhD}$ thesis at the University of Sarajevo working in the Theoretical Physics Group of Prof. Dr. Dejan B. Milošević. The focus of his research is improving existing and developing new numerical methods for solving time-dependent Schrödinger equation for studying interaction of atomic and molecular targets with a strong laser fields with the emphasis on processes such as high-order harmonic generation and above-threshold ionization. He currently works at the Department of Physics at University of Sarajevo as an Assistant Professor.

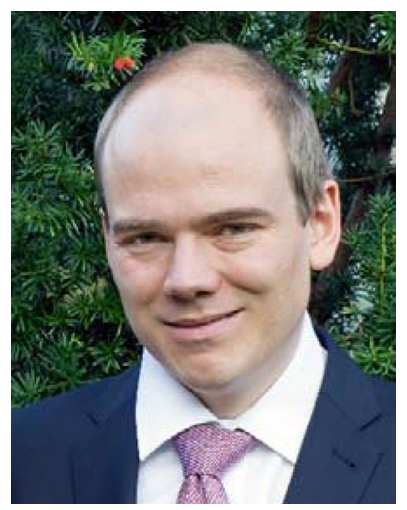

Matthias Kübel received his $\mathrm{PhD}$ from the University of Munich. After a postdoc at the National Research Council of Canada in Ottawa, Ontario, he joined the Institute of Optics and Quantum Electronics (IOQ) at the University of Jena, Germany. In 2020, he was awarded an Emmy Noether grant by the Deutsche Forschungsgemeinschaft and is now setting up a new research group at the IOQ. His research interests include experiments for manipulating quantum dynamics in phase controlled laser fields, and imaging of electronic and nuclear motion in molecules with ultrahigh temporal resolution. 